\title{
Public information and electoral bias
}

\author{
Curtis R. Taylor, Huseyin Yildirim* \\ Department of Economics, Duke University, Box 90097, Durham, NC 27708, United States
}

\section{A R T I C L E I N F O}

\section{Article history:}

Received 10 January 2008

Available online 4 July 2009

\section{JEL classification:}

C72

D72

D82

Keywords:

Costly voting

Public information

Electoral bias

\begin{abstract}
A B S T R A C T
We present a theory of voting that predicts that elections are more likely to be close, and voter turnout is more likely to be high when citizens possess better public information about the composition of the electorate. These findings suggest that providing more information to potential voters about aggregate political preferences (e.g., through preelection polls or expert forecasts) may undermine the democratic process. Our analysis reveals that if the distribution of political preferences is common knowledge, then the unique type-symmetric equilibrium leads to a stark neutrality result in which each alternative is equally likely to win the election. By contrast, when citizens are ignorant about the preference distribution, the majority is more likely to win the election and expected voter turnout is lower. Welfare is, therefore, unambiguously higher when citizens possess less information about the preference distribution.
\end{abstract}

(c) 2009 Elsevier Inc. All rights reserved.

The time has come, I think, to advocate the unspeakable in a forthright and unapologetic manner, and not in the facetious or peripheral way that tradition and circumstance have heretofore demanded. By our rules of procedure, and by any scientific method of counting or reckoning under these rules, the race for the presidency has ended in a flat tie and should be decided by the toss of the coin...

-Stephen Jay Gould, Boston Globe, November 30, 2000.

\section{Introduction}

The U.S. presidential election of 2000 between George W. Bush and Al Gore resulted in a virtual tie. Gore won the national popular election by 532,994 votes or just $0.35 \%$. Bush won the popular election in the decisive state of Florida by 537 votes or just $0.008 \%$. Bush's ultimate margin of victory in the Electoral College was a mere 5 of 537 votes, the thinnest margin since Hayes beat Tilden by a single vote in 1876. While the U.S. presidential contest of 2000 is one of the most notable elections in recent history to result in a virtual tie, it is not the only one. The Washington State gubernatorial race of 2004, for example, was decided by 129 votes in a hand recount of over 2.9 million ballots. ${ }^{1}$ Similarly, the 1997 race for the U.K. House of Commons seat for Winchester resulted in a deadlock, the official count separating the top two candidates by only 2 votes out of 52,198 cast. The two vote margin was disputed and the election was ultimately decided by a courtordered special runoff. ${ }^{2}$ Indeed, as Barone (2006) notes, close elections appear to be an increasingly common part of the

\footnotetext{
Wh thank Han Hong, Matt Jackson, Canice Prendergast and seminar participants at Columbia, Duke, MIT, NYU(Politics), UC-Berkeley, UCLA, and UPenn for helpful comments and suggestions. Taylor's research was supported in part by NSF grant SES-0417737. Any remaining errors are our own.

* Corresponding author.

E-mail addresses: crtaylor@econ.duke.edu (C.R. Taylor), yildirh@econ.duke.edu (H. Yildirim).

1 See http://www.cnn.com/2005/allpolitics/01/12/washington.governor.ap/\#contentarea.

2 See http://news.bbc.co.uk/vote2001/hi/english/voting_system/newsid_1171000/1171887.stm.
} 
political landscape in modern democracies. The very contentious 2006 national elections in Mexico and Italy were each decided by a margin of less than $1 \%$. Also, Canada and Germany have governments headed by leaders of center-right parties that have only a plurality of parliamentary seats that came in ahead of their center-left predecessors by small margins.

In this paper we present a theory of voting that predicts that elections are more likely to be close and voter turnout is more likely to be high when citizens possess better public information about the composition of the electorate. These findings indicate that providing more information to potential voters about aggregate political preferences (e.g., through polls, political stock markets, or expert forecasts) may actually undermine the democratic process. ${ }^{3}$

Our theory is built on a model in which citizens possess private valuations over electoral outcomes and in which voting is costly. ${ }^{4}$ Within this framework we explore two informational regimes, one in which the distribution of political preferences is common knowledge (the informed-voter setting) and one in which citizens are symmetrically ignorant about this distribution (the uninformed-voter setting). In each regime we characterize a unique type-symmetric Bayesian Nash Equilibrium (BNE) in which all citizens randomize between voting for their preferred alternative and abstaining. ${ }^{5}$ We further show that this is the only behavior consistent with a symmetric BNE of the voting game when the population of citizens is sufficiently large.

We compare expected equilibrium outcomes across the two informational environments and find stark differences. First, in the informed-voter setting, the probability that either alternative wins the election under the mixed-strategy BNE equals $\frac{1}{2}$ regardless of the distribution of political preferences or the cost of voting. This neutrality result stems from the fact that individuals who expect to be in the minority vote with higher probability than those who expect to be in the majority in equilibrium. In other words, individuals who expect to be in the minority suffer less from the free-rider problem. Indeed, their lower expected numbers are offset exactly by their greater expected participation, so that the expected equilibrium number of votes for each alternative is the same regardless of the actual distribution of preferences. ${ }^{6}$

In the uninformed-voter setting, however, citizens are not able to base their voting decisions on the distribution of political preferences, since they know only their own types. In fact, given a symmetric common prior over the parameter governing the distribution of tastes, it follows that all citizens vote with the same probability regardless of type. This equilibrium probability of voting is strictly positive, and the majority group, therefore, wins the election with probability strictly greater than $\frac{1}{2}$. Importantly, it is also possible to show that expected equilibrium voter turnout is lower in the uninformedvoter setting. Hence, the uninformed-voter setting gives rise to elections involving higher expected social benefits and lower expected social costs. In other words, when citizens possess less information about the political landscape, elections are both more democratically efficient (the majority is more likely to win) and more economically efficient (fewer resources are expended in the election).

We also investigate whether the uninformed-voter setting continues to yield higher welfare as the electorate size grows. In particular, we present a limit analysis as the number of citizens tends to infinity. In this context, it is possible to show that the equilibrium number of votes for each alternative correspond to independent random variables following Poisson distributions with endogenously determined means. ${ }^{7}$ Armed with this fact, it is straightforward to verify the asymptotic superiority of the uninformed voter setting. Indeed, our strongest result holds in the limit as the number of citizens tends to infinity and the relative cost of voting approaches zero. In this key situation, the alternative favored by the majority wins the election with probability arbitrarily close to 1 when citizens are uninformed but only with probability $\frac{1}{2}$ when they are informed.

Our private-values costly-voting model follows in the tradition of the pioneering works by Ledyard (1984) and Palfrey and Rosenthal $(1983,1985){ }^{8}$ Ledyard (1984) is primarily concerned with formalizing political competition in a setting with rational voting and endogenously determined political alternatives, ${ }^{9}$ whereas Palfrey and Rosenthal (1983, 1985) focus on the issue of equilibrium voter turnout by fixing policy alternatives. ${ }^{10}$

More recent papers in the private-values costly-voting paradigm include Campbell (1999) and Borgers (2004). ${ }^{11}$ Campbell (1999) studies a model in which members of the minority group possess stronger political preferences (higher values or lower costs) than members of the majority. He presents a limit result that is similar in spirit to our neutrality finding in the

\footnotetext{
3 For statistical evidence that polls influence voter turnout, see Sudman (1986) and West (1991). Klor and Winter (2006) present evidence (from experiments and recent U.S. gubernatorial elections) that the publication of poll results may help the majority win when the population is closely divided. They find that members of the majority are most likely to abstain in lopsided electorates. This appears to be what happened in the most stunning upset in U.S. presidential election history when Truman defeated Dewey in 1948 despite trailing in the polls by as much as $15 \%$.

4 Citizens in our private-values model are differentiated by their intrinsic preferences over political alternatives. Hence, we do not study the information aggregation problem that is the focus of common-value models such as Feddersen and Pesendorfer (1997) and Razin (2003).

5 Mixing arises from the assumption that all citizens possess the same cost of voting. As we discuss in the Conclusion, however, our main findings are robust to a 'purified' version of the model in which voting costs are independently and privately drawn from a continuous distribution.

6 The stark nature of this neutrality result arises from the simplifying assumptions underlying our model. A qualitatively similar finding would, however, still obtain in a less stylized but less transparent setting.

7 This finding is reminiscent of Myerson (1998, 2000). There are, however, two important differences. First, the means of the Poisson distributions in our model are determined endogenously. Second, they are finite when voting costs are positive.

8 See, Hansen et al. (1987) for empirical evidence, and Levine and Palfrey (2007) for experimental evidence in favor of this model.

9 For recent work on strategic candidacy, see Dutta et al. (2001). For an intriguing comprehensive study of the origins and persistence of democratic institutions, see Acemoglu and Robinson (2006).

10 Aldrich (1993) surveys the three main theories of voter turnout, including strategic models with costly voting

11 See also Osborne et al. (2000) who investigate strategic costly participation in meetings rather than elections.
} 
Table 1

Ex post payoffs.

\begin{tabular}{lll}
\hline Action/Outcome & $t$ wins & $t$ loses \\
\hline Abstain & 1 & 0 \\
Vote & $1-c$ & $0-c$ \\
\hline
\end{tabular}

informed-voter setting. Specifically, Campbell finds that the minority group wins the election with probability no less than $\frac{1}{2}$ when the number of citizens tends to infinity. However, Campbell does not consider the impact of public information on electoral outcomes or voter turnout.

Borgers (2004) investigates a version of our informed-voter setting in which it is common knowledge that the distribution of political preferences is symmetric (i.e., each citizen is equally likely to prefer either alternative). In this context, Borgers shows that equilibrium voter turnout may be excessive, and he argues cogently that compulsory-voting laws, therefore, may make little sense. Because it focuses on symmetrically distributed types, Borgers' model cannot address the electoral bias at the heart of our analysis. Moreover, Borgers does not consider the uninformed-voter setting and thus the impact of public information on expected turnout or electoral outcomes.

Two recent papers that are perhaps the closest to our investigation are Krasa and Polborn (2009) and Goeree and Grosser (2007). Krasa and Polborn generalize the Borgers (2004) model to incorporate a general distribution of political preferences and a large electorate. They independently derive an asymptotic neutrality result akin to the one we present below in the informed-voter setting, which leads them to recommend subsidizing voter participation. Our findings in the uninformedvoter setting, by contrast, illustrate that political neutrality can be broken with even lower turnout than in the Krasa and Polborn model if voters are uncertain about the distribution of political preferences. Goeree and Grosser (2007) also study the impact of information on electoral outcomes in small elections. Several of their findings regarding the effect of information have analogues in our analysis of small elections, though they were independently discovered in a somewhat different setup. Goeree and Grosser do not, however, investigate large elections - the setting in which our most important findings are demonstrated ${ }^{12}$ - or comparative static and welfare properties of information uncertainty.

In a common-values setting, Martinelli (2006) examines the performance of elections in aggregating information when information, rather than participation, is costly. Martinelli shows that while the information acquired by each voter approaches to zero as the electorate becomes large, it does so slowly enough for the electorate as a whole to (approximately) make the "right" decision. Martinelli's finding parallels our limit result under the uninformed-setting: as the electorate grows, each individual votes with a vanishingly small probability, but the alternative favored by the majority prevails (almost) surely. Finally, Bernhardt et al. (forthcoming) investigate a setting complementary to ours in which candidates learn about voter preferences through polls. They find that citizens prefer private polls to public ones because public polls induce the candidates to cluster their platforms around the preferences of the median voter. Hence, the electorate may be better represented when candidates possess less information about voter preferences.

In the next section we set out the model. Sections 3 and 4 contain the analysis of the informed-voter setting and the uninformed-voter setting respectively. In Section 5 we perform welfare comparisons across the two regimes. Section 6 contains an extension investigating the impact of increased uncertainty on the equilibrium in the uninformed-voter setting. We conclude in Section 7 with a brief discussion of three avenues for possible future research. Several technical lemmas and the proofs of all results appear in Appendix A.

\section{The model}

There are $n \geqslant 2$ agents who may cast a vote in an election between two alternatives, $A$ and $B$. Each agent is one of two types, one who prefers $A$ or one who prefers $B$. A type $t$ agent receives a gross payoff normalized to 1 , if alternative $t$ is implemented and 0 otherwise for $t=A, B .^{13}$ The cost of voting is $c \in(0,1]$ for all agents. ${ }^{14}$ Hence, each agent possesses two (relevant) actions, to abstain or to vote for his preferred alternative because abstaining strictly dominates voting for one's less preferred alternative in this context. The ex post payoff of a type $t$ agent is given in Table 1.

Agents simultaneously choose whether to vote. The election is decided by majority rule and ties are broken by a fair coin toss. Each agent privately knows his type but believes that the preferences of the other agents are determined by realizations of i.i.d. random variables where the probability that an agent is type $t$ is $\lambda_{t} \in(0,1)$ and $\lambda_{A}+\lambda_{B}=1$.

We compare type-symmetric Bayesian-Nash Equilibrium (BNE) outcomes of the class of voting games just described across two informational settings. In the informed-voter setting, the value of $\lambda_{t}$ is common knowledge among all agents. In the uninformed-voter setting, all agents possess a non-degenerate common prior over possible values of $\lambda_{t}$.

\footnotetext{
12 In particular, there always exists an equilibrium in totally mixed strategies if electorate size is sufficiently large.

13 To avoid repetition, whenever we use $t$ and $t^{\prime}$, we mean $t, t^{\prime}=A, B$ and $t \neq t^{\prime}$ throughout.

14 Given that the benefit of winning is normalized to $1, c$ represents the cost-to-benefit ratio. In the Conclusion, we briefly discuss the robustness of our results with respect to heterogenous costs of voting.
} 


\section{Informed voters}

Let $\phi_{t} \in[0,1]$ be the probability that a type- $t$ agent votes (i.e., he abstains with probability $1-\phi_{t}$ ). A symmetric BNE in this setting is a pair of probabilities $\left(\phi_{A}, \phi_{B}\right)$ such that it is optimal for a type $t$ agent to vote with probability $\phi_{t}$ when all other agents adhere to this strategy. To find such an equilibrium, note that a type $t$ agent compares the expected payoff from voting, $U_{t}^{1}$, to the expected payoff from abstaining, $U_{t}^{0}$, whose expressions are provided in Appendix A. Let $\Delta_{t}=U_{t}^{1}-U_{t}^{0}$ denote the net expected utility for a type $t$ agent from voting.

In order to write $\Delta_{t}$ in a more useful form, let the ex ante probability that a type $t$ agent votes be denoted by $\alpha_{t}=\lambda_{t} \phi_{t}$. Hence, the ex ante probability of abstaining is $1-\alpha_{A}-\alpha_{B}$. Also, recall that the number of ways $k_{A}$ agents can vote for $A$, $k_{B}$ can vote for $B$, and $n-1-k_{A}-k_{B}$ can abstain is given by the trinomial coefficient $\left(\begin{array}{c}n-1 \\ k_{A}, k_{B}, n-1-k_{A}-k_{B}\end{array}\right)=\frac{(n-1) !}{k_{A} ! k_{B} !\left(n-1-k_{A}-k_{B}\right) !}$.

Lemma 1. Suppose $0<\phi_{t}<1$ for $t=A, B$. Then, the net expected utility to a type $t$ agent from voting can be written

$$
\Delta_{t}=\frac{1}{2} P_{t}\left(\alpha_{t}, \alpha_{t^{\prime}}, n\right)-c
$$

where

$$
\begin{aligned}
P_{t}\left(\alpha_{t}, \alpha_{t^{\prime}}, n\right) \equiv & \sum_{k=0}^{\left\lfloor\frac{n-1}{2}\right\rfloor}\left(\begin{array}{c}
n-1 \\
k, k, n-1-2 k
\end{array}\right) \alpha_{t}^{k} \alpha_{t^{\prime}}^{k}\left(1-\alpha_{t}-\alpha_{t^{\prime}}\right)^{n-1-2 k} \\
& +\sum_{k=0}^{\left\lfloor\frac{n-2}{2}\right\rfloor}\left(\begin{array}{c}
n-1 \\
k, k+1, n-2-2 k
\end{array}\right) \alpha_{t}^{k} \alpha_{t^{\prime}}^{k+1}\left(1-\alpha_{t}-\alpha_{t^{\prime}}\right)^{n-2-2 k},
\end{aligned}
$$

for $t=A, B, t \neq t^{\prime}$, and $\lfloor\cdot\rfloor$ is the usual operator that rounds a number to the lower integer when necessary.

This lemma has a very intuitive interpretation. It says that the net expected utility to a type $t$ agent from voting is composed of three parts. Specifically, $P_{t}\left(\alpha_{t}, \alpha_{t^{\prime}}, n\right)$ is the ex ante probability that his vote is pivotal. The first summation in (2) is the probability that his vote breaks a tie (i.e., the event that $k$ of the other agents vote for each alternative and $n-1-2 k$ of them abstain). The second summation is the probability that his vote creates a tie (i.e., the event that $k$ of the other agents vote for alternative $t, k+1$ vote for $t^{\prime}$, and $n-2-2 k$ abstain). In each case, the agent's vote raises the probability of winning by $\frac{1}{2}$. The third term in Eq. (1) is simply the agent's cost of voting. Note that we restrict attention to totally mixed strategies, but we will show that this is the case for sufficiently large electorates and/or a sufficiently large cost of voting.

A convenient feature of Lemma 1 is that the net expected utility from voting can be expressed entirely in terms of the ex ante probabilities, $\alpha_{A}$ and $\alpha_{B}$. This derives from the fact that $\lambda_{A}$ and $\lambda_{B}$ are common knowledge so that each agent perfectly customizes his voting strategy to the distribution of political preferences. This along with the symmetry of the net-expected payoff functions given in Eq. (1) yield

Proposition 1 (Weak neutrality in small elections). Suppose $\left(\phi_{A}, \phi_{B}\right)$ is a symmetric BNE in totally mixed strategies; i.e., $0<\phi_{t}<1$ for $t=A, B$.

(a) The ex ante probability that an agent votes for alternative A equals the ex ante probability that he votes for $B$,

$$
\lambda_{A} \phi_{A}=\lambda_{B} \phi_{B}
$$

(b) Both political outcomes are equally likely in equilibrium,

$$
\operatorname{Pr}\left\{t \text { wins } \mid \lambda_{t}\right\}=\frac{1}{2}
$$

This result says that in a type-symmetric mixed-strategy BNE, the probability that either alternative is implemented does not depend on $\lambda_{t}$. Hence, the alternative that is preferred by the expected majority wins the election with the same probability as the one that is preferred by the expected minority. The intuition underlying this result rests on a delicate trade-off between an agent's incentive to win the election and his incentive to free ride on other voters who likely share his preferences. For instance, if $\lambda_{A}>\frac{1}{2}$, then a type $A$ agent knows that he is most likely a member of the majority and he has a relatively high incentive to free ride (i.e., he votes with lower probability). On the other hand, a type $B$ agent knows that he is most likely a member of the minority and he has a relatively low incentive to free ride (i.e., he votes with higher probability). Remarkably, these effects exactly balance in equilibrium leading to a situation in which the inherently stronger position of a type $A$ agent is completely neutralized. ${ }^{15}$

\footnotetext{
15 Anecdotal evidence supporting Proposition 1 comes from the frequent failure of pre-election polls in predicting outcomes. For instance, Jowell et al. (1993) report that in the 1992 British election, while all polls predicted a very close finish, with the Labor Party victory, the Conservatives in fact won with
} 
In light of Proposition 1, let $\alpha$ denote the ex ante probability that an agent votes for one of the two alternatives in a symmetric totally mixed-strategy BNE (when one exists). Since the ex ante probability that he votes at all is $2 \alpha$, it must be that $0<\alpha \leqslant \frac{1}{2}$. In order to find a mixed-strategy equilibrium, it is, therefore, necessary to find a solution in this range to the polynomial indifference equation

$$
\frac{1}{2} P(\alpha, n)-c=0
$$

where $P(\alpha, n)=P_{t}(\alpha, \alpha, n)$.

The function $P(\alpha, n)$ gives the probability that a given agent's vote is pivotal when the other $n-1$ agents vote for each alternative with ex ante probability $\alpha$ and abstain with probability $1-2 \alpha$.

Lemma 2. $P(\alpha, n)$ is strictly decreasing in $\alpha \in\left[0, \frac{1}{2}\right]$.

Intuitively, a given agent's vote is less likely to be pivotal when others vote with a higher ex ante probability leading to a greater expected voter turnout. An important implication of Lemma 2 is that there is at most one solution to Eq. (3). Furthermore, there exists a solution if and only if $\frac{1}{2} P(0, n)>c$ and $\frac{1}{2} P\left(\frac{1}{2}, n\right) \leqslant c$. Let $\alpha^{*}(c, n)$ denote the solution when it exists. Note that existence of $\alpha^{*}(c, n)$ is necessary but not sufficient for existence of a totally mixed-strategy BNE. In particular, existence of a totally mixed-strategy BNE also requires $0<\phi_{t}=\alpha^{*}(c, n) / \lambda_{t}<1$.

Proposition 2 (Characterization). There exists a unique type-symmetric BNE in totally mixed strategies if and only if

$$
\underline{c}(n)<c<\frac{1}{2}
$$

and

$$
\alpha^{*}(c, n)<\lambda_{t}<1-\alpha^{*}(c, n)
$$

where

$$
\underline{c}(n) \equiv \begin{cases}\left(\begin{array}{c}
n-1 \\
\frac{n-1}{2}
\end{array}\right)\left(\frac{1}{2}\right)^{n}, & \text { if } n \text { is odd, } \\
\left(\begin{array}{c}
n-1 \\
\frac{n}{2}
\end{array}\right)\left(\frac{1}{2}\right)^{n}, & \text { if } n \text { is even. }\end{cases}
$$

Condition (4) specifies the range of voting costs for which a solution $\alpha^{*}(c, n) \in\left(0, \frac{1}{2}\right]$ to (3) exists. Specifically, if $c \geqslant \frac{1}{2}$, then the unique BNE is for all agents to abstain. A deviating agent would surely be pivotal $(P(0, n)=1)$, but deviating is still not profitable $\left(\frac{1}{2} P(0, n) \leqslant c\right)$. On the other hand, if $c \leqslant \underline{c}(n)$, then at least one type of agent must vote with probability 1 in equilibrium since $\alpha^{*}(c, n) \geqslant \frac{1}{2}$. When (4) is satisfied, existence of a symmetric totally mixed-strategy BNE also requires that $\lambda_{t}$ not be too extreme. In particular, if (5) fails, then the agents in the expected minority will be unable to "neutralize" the expected majority even if they vote with certainty.

As an illustration of Proposition 2, suppose $n=2$. In this case $\alpha^{*}=1-2 c$. Hence, a type-symmetric mixed-strategy equilibrium exists if and only if $c \in\left(\frac{1}{4}, \frac{1}{2}\right)$ and $\lambda_{t} \in(1-2 c, 2 c)$. Next, we show that such an equilibrium exists for a wider range of parameters as the electorate size increases.

Electorate size and the prevalence of the neutrality. Our objective here is to perform a comparative static with respect to the electorate size, $n$, and do a limit analysis for $n \rightarrow \infty$. Since we are ultimately interested in social welfare, we characterize not only the election outcome but also the expected turnout in the limit. We emphasize, however, that our limit analysis is meant to present a theoretical benchmark rather than to resolve the celebrated "paradox of not voting" formalized by Palfrey and Rosenthal (1985).

\section{Lemma 3.}

(i) $\alpha^{*}(c, n)$ is decreasing in $n$, and converges to 0 as $n \rightarrow \infty$.

(ii) $\underline{c}(n)$ is decreasing, and converges to 0 as $n \rightarrow \infty$.

(iii) $P\left(\alpha_{t}^{*}(c, n), \alpha_{t^{\prime}}^{*}(c, n), n\right)=2 c$ as $n \rightarrow \infty$.

a significant lead of 7.6 percent. Similarly, Durand et al. (2001) record that in the 1998 general elections in Quebec, public polls overwhelmingly predicted an easy victory for the ruling Parti Quebecois, a party dedicated to Quebec sovereignty. Yet, the Quebec Liberal Party ended up winning. Finally, Durand et al. (2004) note that in the 2002 French presidential elections, although polls consistently predicted a matchup between the incumbent president, Jacques Chirac, and the incumbent prime minister, Lionel Jospin, in the second round, Jean-Marie Le Pen from an extremist right-wing party instead finished second. Interestingly, a common defense by the pollsters has been the weak turnout by the expected majority on the election day. 
Part (i) of Lemma 3 reveals that as the electorate size increases, each agent is less likely to vote. This is because a larger electorate means a proportionally larger minority group and a proportionally larger majority group, which, in turn, means a more severe free rider problem within each group. Thus, it must take an even smaller cost for an agent to vote with certainty, as recorded in part (ii). Together with Proposition 2, parts (i) and (ii) imply that a type-symmetric equilibrium in totally mixed strategies, and therefore the weak neutrality identified in Proposition 1, is more likely to obtain, as the number of citizens grows. Finally, since each agent plays a mixed strategy in equilibrium in a sufficiently large election, part (iii) states that the equilibrium indifference condition in (3) must hold. ${ }^{16}$

Next, we determine the expected turnout in the limit. To do so, let $X_{t}$ be the number of votes for alternative $t$, and $X_{0}=n-X_{A}-X_{B}$ be the number of abstentions. Using this notation, a type $t$ agent's vote is pivotal if and only if $X_{t}=X_{t^{\prime}}$ (his vote creates a tie), or $X_{t}=X_{t^{\prime}}+1$ (his vote breaks a tie). Hence, the probability that his vote is pivotal can be written

$$
P\left(\alpha_{t}^{*}(c, n), \alpha_{t^{\prime}}^{*}(c, n), n\right)=\operatorname{Pr}\left\{X_{t}=X_{t^{\prime}}\right\}+\operatorname{Pr}\left\{X_{t}=X_{t^{\prime}}+1\right\} .
$$

Now, observe that $\left(X_{A}, X_{B}, X_{0} \mid n\right) \sim \operatorname{Multinomial}\left(\alpha_{A}^{*}(c, n), \alpha_{B}^{*}(c, n), 1-\alpha_{A}^{*}(c, n)-\alpha_{B}^{*}(c, n) \mid n\right)$. Although, for a fixed $n$, the random variables, $X_{A}$ and $X_{B}$, are clearly correlated, ${ }^{17}$ the following lemma shows they are independent as $n \rightarrow \infty$. Let $m_{t}=\lim _{n \rightarrow \infty}\left[n \alpha_{t}^{*}(c, n)\right]$ be the expected equilibrium turnout for type $t$ citizens in the limit. ${ }^{18}$

Lemma 4. The limiting marginal distributions of $X_{A}$ and $X_{B}$ are independent Poisson distributions with means $m_{A}$ and $m_{B}$, respectively. Hence, the limiting distribution of $X_{A}+X_{B}$ is also Poisson with mean $m_{A}+m_{B}$.

In light of Lemma 4 , let $f(k \mid \mu)$ be the p.d.f. for a Poisson distribution with mean $\mu$. Recall that $f(k \mid \mu)=\frac{\mu^{k} e^{-\mu}}{k !}$ for $k=0,1, \ldots$ Combining (7) and Lemma 4 , it follows that

$$
\begin{aligned}
\lim _{n \rightarrow \infty} P_{t}\left(\alpha_{t}^{*}(n), \alpha_{t^{\prime}}^{*}(n), n\right) & =\lim _{n \rightarrow \infty}\left[\operatorname{Pr}\left\{X_{t}=X_{t^{\prime}}\right\}+\operatorname{Pr}\left\{X_{t}=X_{t^{\prime}}+1\right\}\right] \\
& =\sum_{k=0}^{\infty} f\left(k \mid m_{t}\right) f\left(k \mid m_{t^{\prime}}\right)+\sum_{k=0}^{\infty} f\left(k \mid m_{t}\right) f\left(k+1 \mid m_{t^{\prime}}\right) \\
& \equiv \Phi^{I}\left(m_{t}, m_{t^{\prime}}\right) .
\end{aligned}
$$

Moreover, using part (iii) of Lemma 3, Eq. (8) implies that the equilibrium limiting turnouts (in the Informed regime), $m_{A}^{I}$ and $m_{B}^{I}$, must solve

$$
\Phi^{I}\left(m_{A}, m_{B}\right)=2 c \text { and } \Phi^{I}\left(m_{B}, m_{A}\right)=2 c .
$$

Proposition 3 (Strong neutrality in large elections). In the informed regime, for any voting cost $c \in\left(0, \frac{1}{2}\right)$ and any distribution of preferences $\lambda_{t} \in(0,1)$, there exists a critical population size $\bar{n}$ such that $n \geqslant \bar{n}$ implies the existence of a unique type-symmetric BNE. Moreover,

(a) $\lim _{n \rightarrow \infty} \operatorname{Pr}\left\{\right.$ Alternative $t$ wins $\left.\mid \lambda_{t}\right\}=\frac{1}{2}$.

(b) $m_{A}^{I}=m_{B}^{I}=\frac{M^{I}}{2}$, where the expected aggregate turnout, $M^{I} \in(0, \infty)$, uniquely solves $\Phi^{I}\left(\frac{M}{2}, \frac{M}{2}\right)=2 c$.

Part (a) of Proposition 3 is a direct implication of Lemma 3. It indicates that the neutrality result identified in Proposition 1 is endemic to elections with large populations. In other words, when the distribution of voter preferences is common knowledge, large elections are likely to be close for (approximately) the whole parameter space. As explained in Proposition 1, this neutrality result is a consequence of the strategic voting behavior: when agents have "good" information about voter preferences, they are able to customize their voting decisions to the relative size of the groups.

Part (b) of Proposition 3 points to an additional kind of neutrality, which is absent in small elections. It says that in large elections, the expected turnout for each group is independent of the distribution of voter preferences. ${ }^{19}$ That is, two large elections with $\lambda_{t}=\frac{1}{2}$ and $\lambda_{t}=\frac{99}{100}$ result in the same expected turnout for each alternative, contrary to what intuition suggests.

\footnotetext{
16 Note that by (3), $P\left(\alpha_{t}^{*}(c, n), \alpha_{t^{\prime}}^{*}(c, n), n\right)=2 c$ for all $n$ at a totally mixed-strategy equilibrium, and by Lemma 3 , such an equilibrium is the only one as $n \rightarrow \infty$.

17 The correlation coefficient is $-n \alpha^{* 2}(c, n)$.

18 It can be easily verified that these limits exist and they are finite.

19 Of course, the equality of turnouts is an artifact of the assumption that the cost of voting is the same for both types of agents. However, it should be clear from (9) that even if costs were different, the limit turnouts would still be independent of the distribution of political preferences, i.e., $\lambda_{t}$. Moreover, this result is robust to an extension with private cost of voting, where each agent draws his cost from a distribution. For more on this point, see the discussion in Section 7 and in Taylor and Yildirim (2008) for a formal derivation.
} 
Remark 1. While theoretically intriguing, by the weak and strong neutrality results, we do not suggest that elections, especially those with large electorates, will tend to be close regardless of the actual polling results; because in reality, polls provide only a noisy estimate of the preference distribution, $\lambda_{t}$. Such noise is likely to prevent citizens from perfectly customizing their voting decisions to the relative size of the political groups. In fact, we show in the next section that when citizens are uncertain about the preference distribution, the neutrality result breaks and it does so in favor of the majority.

Remark 2. One may wonder whether our restriction to type-symmetric strategies may be unduly intensifying the free-rider problem and preventing the majority from winning. Note, however, that in the presence of the incomplete information about others' preferences, and the resulting uncertainty about the group sizes, perfectly coordinated voting strategies for the majority to guarantee a win would not be sustained in any equilibrium.

\section{Uninformed voters}

Consider a setting in which $\lambda_{t}$ is not common knowledge. Specifically, suppose that before learning their types, the agents' beliefs about $\lambda_{t}$ correspond to a non-degenerate common prior distribution. For ease of exposition, assume that the prior is symmetric and defined over a finite set of values, $\lambda^{1}>\lambda^{2}>\cdots>\lambda^{r}$, where $r \geqslant 2 .{ }^{20}$ Symmetry of the prior requires $\operatorname{Pr}\left\{\lambda_{t}=\lambda^{i}\right\}=\operatorname{Pr}\left\{\lambda_{t^{\prime}}=\lambda^{i}\right\}=\theta^{i} \in(0,1)$, for $i=1, \ldots, r$. This implies that $E\left[\lambda_{t}\right]=\frac{1}{2}$. Also, after learning their types, agents' updated beliefs are

$$
\operatorname{Pr}\left\{\lambda_{t}=\lambda^{i} \mid t\right\}=2 \theta^{i} \lambda^{i}
$$

Notice $\operatorname{Pr}\left\{\lambda_{t}=\lambda^{i} \mid t\right\} \geqslant \operatorname{Pr}\left\{\lambda_{t}=\lambda^{i}\right\}$ if and only if $\lambda^{i} \geqslant \frac{1}{2}$. That is, upon observing their own types, agents put more weight on being in the majority - a fact that will be useful in developing the intuition below.

As in the informed-voter setting, a symmetric BNE in this context corresponds to a pair of voting probabilities $\left(\phi_{A}, \phi_{B}\right)$. Moreover, $\left(\phi_{A}, \phi_{B}\right)$ is a symmetric BNE in totally mixed strategies if and only if it satisfies the indifference conditions

$$
\frac{1}{2} E\left[P_{t}\left(\lambda_{t} \phi_{t}, \lambda_{t^{\prime}} \phi_{t^{\prime}}\right) \mid t\right]-c=0,
$$

where $P_{t}(\cdot, \cdot)$ is defined in (2).

Proposition 4 (Non-neutrality). Suppose $\left(\phi_{A}, \phi_{B}\right)$ is a type-symmetric BNE in totally mixed strategies. Then,

(a) $\phi_{A}=\phi_{B}=\phi$.

(b) For $n<\infty$, $\operatorname{Pr}\left\{t\right.$ wins $\left.\mid \lambda_{t}=\lambda^{i}\right\}>\frac{1}{2}$ if and only if $\lambda^{i}>\frac{1}{2}$.

Part (a) of this result indicates that any type-symmetric BNE in totally mixed strategies must be strongly symmetric in the sense that all agents vote with the same probability regardless of type. ${ }^{21}$ The intuition is straightforward. When the prior is symmetric, an agent who discovers that he is type $A$ learns as much about the environment as an agent who discovers that he is type $B$. Hence, both types vote with the same probability in equilibrium. This contrasts sharply with part (a) of Proposition 1 where it is shown that agents in the expected minority vote with higher probability than those in the expected majority. This effect is absent in the uninformed-voter setting because no agent expects to be in the minority conditional on learning only his own type. In particular, $E\left[\lambda_{t} \mid t\right]=2 E\left[\lambda_{t}^{2}\right]>\frac{1}{2}$.

Next, consider part (b). Because both types of agents vote with the same probability, it follows that the alternative with the expected majority is strictly more likely to win the election. Again, this contrasts with Proposition 1 where it was shown that each alternative was equally likely to win when $\lambda_{t}$ was common knowledge.

In order to characterize a symmetric BNE in totally mixed strategies in the uninformed-voter setting, we use $\phi_{A}=\phi_{B}=\phi$ and note that the expected probability of being pivotal in equilibrium is

$$
Q(\phi, n) \equiv E\left[P_{t}\left(\lambda_{t} \phi,\left(1-\lambda_{t}\right) \phi, n\right) \mid t\right]=\sum_{i=1}^{\left\lfloor\frac{r+1}{2}\right\rfloor} 2 \theta^{i} T\left(\phi, n \mid \lambda_{A}=\lambda^{i}\right)
$$

where we define

$$
T\left(\phi, n \mid \lambda_{A}=\lambda^{i}\right) \equiv \lambda^{i} P_{A}\left(\lambda^{i} \phi,\left(1-\lambda^{i}\right) \phi, n \mid A\right)+\left(1-\lambda^{i}\right) P_{B}\left(\left(1-\lambda^{i}\right) \phi, \lambda^{i} \phi, n \mid B\right)
$$

\footnotetext{
20 Symmetry of the prior is assumed both for tractability and in order to isolate the role of information from any ex ante advantage. It is worth emphasizing, however, that the welfare results presented below are conditioned on the realization of $\lambda^{i}$; i.e., we do not assume that actual political preferences are symmetrically distributed.

21 This strong symmetry result, which Goeree and Grosser (2007) simply assume, will play a key role in the main finding about welfare.
} 


$$
\begin{aligned}
= & \sum_{k=0}^{\left\lfloor\frac{n-1}{2}\right\rfloor}\left(\begin{array}{c}
n-1 \\
k, k, n-1-2 k
\end{array}\right)\left(\lambda^{i}\left(1-\lambda^{i}\right)\right)^{k} \phi^{2 k}(1-\phi)^{n-1-2 k} \\
& +2 \sum_{k=0}^{\left\lfloor\frac{n-2}{2}\right\rfloor}\left(\begin{array}{c}
n-1 \\
k, k+1, n-2-2 k
\end{array}\right)\left(\lambda^{i}\left(1-\lambda^{i}\right)\right)^{k+1} \phi^{2 k+1}(1-\phi)^{n-2-2 k} .
\end{aligned}
$$

Hence, the equilibrium $\phi^{*}$ must solve

$$
\frac{1}{2} Q(\phi, n)-c=0 \text {. }
$$

Proposition 5. In the uninformed setting, there exists a unique type-symmetric BNE in totally mixed strategies if and only if $\underline{\underline{c}}(n)<$ $c<\frac{1}{2}$, where

$$
\underline{\underline{c}}(n)= \begin{cases}\underline{c}(n) 2^{n} E\left[\lambda_{t}^{\frac{n+1}{2}}\left(1-\lambda_{t}\right)^{\frac{n-1}{2}}\right], & \text { if } n \text { is odd, } \\ \underline{c}(n) 2^{n} E\left[\left(\lambda_{t}\left(1-\lambda_{t}\right)\right)^{\frac{n}{2}}\right], & \text { if } n \text { is even. }\end{cases}
$$

Similar to Proposition 2 of the preceding section, this result characterizes the region of the parameter space where the unique type-symmetric BNE in totally mixed strategies obtains. Specifically, if $c \geqslant \frac{1}{2}$, then no agents vote in equilibrium, and if $c \leqslant \underline{c}(n)$, then they all vote with certainty. In order to facilitate comparison with the informed-voter setting, it is necessary to know how $\underline{\underline{c}}(n)$ compares with $\underline{\underline{c}}(n)$.

Lemma 5. For any $n, \underline{\underline{c}}(n)<\underline{c}(n)$.

Lemma 5 indicates that if a type-symmetric BNE in totally mixed strategies obtains in the informed-voter setting, then an analogous BNE obtains in the uninformed-voter setting as well. This is because agents are more likely to free ride and follow a mixed strategy when they are in the majority. In the uninformed regime, this is exactly what all agents think after updating their beliefs about $\lambda_{t}$ based on their own types.

Next, we consider the large election properties of the uninformed regime.

Electorate size and the majority advantage. The analysis in this section essentially mimics that presented for the informed regime. We first note the following intuitive observation regarding the probability of voting.

Lemma 6. $\phi^{*}(n)$ strictly decreases in $n$ and converges to 0 , as $n \rightarrow \infty$.

That is, the equilibrium probability of voting decreases in the electorate size and becomes negligible in large elections, because the free-rider problem is exacerbated in each political group.

Now, recall that $X_{t}$ and $X_{0}$ denote the number of votes for alternative $t$, and the number of abstentions, respectively. It is clear that $\left(X_{A}, X_{B}, X_{0} \mid n\right) \sim \operatorname{Multinomial}\left(\lambda_{A} \phi^{*}(n), \lambda_{B} \phi^{*}(n), 1-\phi^{*}(n) \mid n\right)$. The following result parallels Lemma 4.

Lemma 7. The limiting marginal distributions of $X_{A}$ and $X_{B}$ are independent Poisson distributions with respective means $\lambda_{A} M$ and $\lambda_{B} M$, where $M=\lim _{n \rightarrow \infty}\left[n \phi^{*}(n)\right]$.

We now re-write the pivot probability in (11) in terms of $X_{A}$ and $X_{B}$. To do so, we first observe that

$$
T\left(\phi^{*}(n), n \mid \lambda_{A}=\lambda^{i}\right)=\operatorname{Pr}\left\{X_{B}=X_{A}\right\}+2 \lambda^{i} \operatorname{Pr}\left\{X_{B}=X_{A}+1\right\} .
$$

Hence, given the respective means, $\lambda^{i} M$ and $\left(1-\lambda^{i}\right) M$ for alternative $A$ and $B$ for $\lambda_{A}=\lambda^{i}$, we have

$$
\begin{aligned}
\lim _{n \rightarrow \infty} Q\left(\phi^{*}(n), n\right) & =\sum_{i=1}^{\left\lfloor\frac{r+1}{2}\right\rfloor} 2 \theta^{i} \lim _{n \rightarrow \infty}\left[\operatorname{Pr}\left\{X_{B}=X_{A}\right\}+2 \lambda^{i} \operatorname{Pr}\left\{X_{B}=X_{A}+1\right\}\right] \\
& =\sum_{i=1}^{\left\lfloor\frac{r+1}{2}\right\rfloor} 2 \theta^{i}\left[\sum_{k=0}^{\infty} f\left(k \mid \lambda^{i} M\right) f\left(k \mid\left(1-\lambda^{i}\right) M\right)+2 \lambda^{i} \sum_{k=0}^{\infty} f\left(k \mid \lambda^{i} M\right) f\left(k+1 \mid\left(1-\lambda^{i}\right) M\right)\right] \\
& \equiv \Phi^{U}(M \mid \theta, \lambda) .
\end{aligned}
$$

From (13), the expected aggregate turnout, $M^{U}$, is determined by

$$
\Phi^{U}(M \mid \theta, \lambda)=2 c .
$$


Proposition 6. In the uninformed regime, for any voting cost $c \in\left(0, \frac{1}{2}\right)$ and any distribution of preferences $(\lambda, \theta)$, there exists a critical population size $\bar{n}$ such that $n \geqslant \bar{n}$ implies the existence of a unique symmetric BNE. Moreover,

(a) $\lim _{n \rightarrow \infty} \operatorname{Pr}\left\{t\right.$ wins $\left.\mid \lambda_{t}=\lambda^{i}\right\}>\frac{1}{2}$ if and only if $\lambda^{i}>\frac{1}{2}$.

(b) $\lim _{c \rightarrow 0} \lim _{n \rightarrow \infty} \operatorname{Pr}\left\{t\right.$ wins $\left.\mid \lambda_{t}=\lambda^{i}\right\}=1$ if and only if $\lambda^{i}>\frac{1}{2}$.

(c) $m_{A}^{U}=\lambda_{A} M^{U}$, and $m_{B}^{U}=\lambda_{B} M^{U}$, where the aggregate turnout, $M^{U} \in(0, \infty)$, uniquely solves (15).

Part (a) of Proposition 6 indicates that the non-neutrality result identified in Proposition 4 remains valid for large elections. The reason is that while the probability of voting becomes negligible, the aggregate turnout is still significant, and without electoral bias under the uninformed regime, large elections favor the majority. In fact, as the cost of voting converges to zero, the aggregate turnout grows so large that the majority wins with virtual certainty, which is recorded in part (b). The last part of Proposition 6 simply says that the expected turnout by each type of citizen is proportional to the fraction of that type in the population. This contrasts with the informed case where the expected turnout is equal across groups regardless of the initial distribution of preferences.

Before proceeding to the welfare analysis, it is worth comparing part (b) of Proposition 6 to Martinelli (2006). As mentioned in Section 1, Martinelli studies a common-values model of voting in which it is the information, rather than participation, that is costly. He finds that while the amount of information each voter gathers becomes negligible as the number of citizens increases, it does so slowly enough that the electorate remains well informed to choose the "right" alternative.

\section{Welfare comparison}

Armed with the equilibrium outcomes of the two informational regimes, we are now ready to investigate their implications for social welfare. The expected social welfare in this context is the difference between the expected social benefit and the expected social cost. Given the payoff structure in Table 1, and conditional on $\lambda_{A}=\lambda^{i}$, the expected social benefit is $B\left(\phi, n \mid \lambda_{A}=\lambda^{i}\right)=\operatorname{Pr}\left\{A\right.$ wins $\left.\mid \lambda^{i}\right\} \lambda^{i} n+\operatorname{Pr}\left\{B\right.$ wins $\left.\mid 1-\lambda^{i}\right\}\left(1-\lambda^{i}\right) n$, whereas the expected social cost is $C\left(\phi, n \mid \lambda_{A}=\lambda^{i}\right)=$ $c \lambda^{i} \phi_{A}^{*} n+c\left(1-\lambda^{i}\right) \phi_{B}^{*} n$. Hence, the expected social welfare is $W\left(\phi, n \mid \lambda_{A}=\lambda^{i}\right)=B\left(n \mid \lambda_{A}=\lambda^{i}\right)-C\left(n \mid \lambda_{A}=\lambda^{i}\right)$. From an ex ante point of view however, since the realization of $\lambda_{A}$ is unknown, we compare the ex ante social benefit, cost and welfare for a given $\theta$, which we write as

$$
\begin{aligned}
& \bar{B}(\theta, \lambda, n)=\sum_{i=1}^{r} \theta^{i} B\left(\phi, n \mid \lambda_{A}=\lambda^{i}\right), \\
& \bar{C}(\theta, \lambda, n)=\sum_{i=1}^{r} \theta^{i} C\left(\phi, n \mid \lambda_{A}=\lambda^{i}\right), \\
& \bar{W}(\theta, \lambda, n)=\sum_{i=1}^{r} \theta^{i} W\left(\phi, n \mid \lambda_{A}=\lambda^{i}\right) .
\end{aligned}
$$

The following result lays out a central finding of this paper.

Proposition 7 (Welfare). Suppose (4) and (5) hold so that a type-symmetric totally-mixed strategy BNE obtains in either informational setting. Then, for all $n \leqslant \infty$

(a) $\bar{C}^{I}(\theta, \lambda, n)>\bar{C}^{U}(\theta, \lambda, n)$,

(b) $\bar{B}^{I}(\theta, \lambda, n)<\bar{B}^{U}(\theta, \lambda, n)$,

(c) $\bar{W}^{I}(\theta, \lambda, n)<\bar{W}^{U}(\theta, \lambda, n)$.

Part (a) reveals that the expected social cost of voting is strictly greater under the informed regime, which is equivalent to saying that the expected turnout is higher. The intuition behind this result is easily grasped. For any $\lambda_{t}$, the expected equilibrium voter turnout is $2 \alpha^{*} n$ in the informed-voter setting and $\phi^{*} n$ in the uninformed-voter setting. To see that $2 \alpha^{*}>$ $\phi^{*}$, consider a situation in which agents vote with the same ex ante probability, $2 \alpha=\phi$, in each setting. In this case, a voter in the informed situation has a higher probability of being pivotal than one in the uninformed situation, $P(\alpha, n)>Q(\phi, n)$. The reason is that when agents have better information, they are better positioned to vote strategically. Specifically, with better information, agents who expect to be in the minority vote with higher probability and agents who expect to be in the majority vote with lower probability. This voter-composition effect leads to a closer election and higher probability of being pivotal in the informed-voter setting when the ex ante probability of voting is the same across regimes. Of course, in equilibrium the probability of being pivotal must equal $2 c$ in both settings. This, however, requires agents to vote with higher ex ante probability in the informed-voter setting, $2 \alpha^{*}>\phi^{*}$. 
Part (b) of Proposition 7 says that the ex ante social benefit is strictly higher in the uninformed regime than in the informed one. The expected social benefit is maximized when the alternative that is preferred by the majority group wins. As explained above, this is more likely to be the case with the uninformed regime. In particular, in a large election with a small cost of voting, the majority wins with probability close to 1 under the uninformed regime whereas this probability still remains at $\frac{1}{2}$ under the informed regime. Together with parts (a) and (b), it follows that expected welfare is unambiguously higher when agents are uninformed about the composition of the electorate, as recorded in part (c).

An implication of this finding is that the public release of information that resolves all uncertainty about the distribution of voter preferences reduces welfare. The reason for this inefficiency is twofold. First, when the distribution of voter preferences becomes common knowledge, each agent knows exactly whether he belongs to the expected minority or the expected majority group. In this instance, as explained following Proposition 1, each agent tailors his voting strategy to his expected group size so that in equilibrium each group is equally likely to win the election. When no information about the distribution of voter preferences is provided, however, each agent votes with the same equilibrium probability, making the expected majority more likely to win, which is obviously better for welfare. Second, as Proposition 7 reveals, the expected total cost of voting is smaller when voters are uninformed. The reason is that when uninformed, all agents put more weight on being in the majority and hence possess less incentive to vote.

\section{An extension: Uncertainty and welfare}

To this point we have compared two informational regimes, and showed that the uninformed regime outperforms the informed one in expected social welfare. Under the uninformed regime, agents are ignorant about whether they belong to the majority or minority. Although this uncertainty leads them to vote less vigorously and keep social cost lower than under the informed regime, it also reduces electoral bias and increases the probability that the majority wins. Staying within the uninformed regime, a natural question is then to ask whether greater uncertainty results in higher social welfare. To investigate this issue, we first define the degree of uncertainty via a mean-preserving spread over $\theta$.

Definition (Mean-preserving spread). Let $\theta^{\prime}=\left(\theta^{1 \prime}, \ldots, \theta^{\left\lfloor\frac{r+1}{2}\right\rfloor \prime}, \ldots, \theta^{r \prime}\right)$ and $\theta=\left(\theta^{1}, \ldots, \theta^{\left\lfloor\frac{r+1}{2}\right\rfloor}, \ldots, \theta^{r}\right)$ be two symmetric distributions over $\left\{\lambda^{1}, \ldots, \lambda^{\left\lfloor\frac{r+1}{2}\right\rfloor}, \ldots, \lambda^{r}\right\}$. Distribution $\theta^{\prime}$ is said to be a mean-preserving spread of $\theta$ if there is some $i_{0} \in\left\{1, \ldots,\left\lfloor\frac{r+1}{2}\right\rfloor\right\}$ such that

$$
\begin{cases}\theta^{i \prime} \geqslant \theta^{i}, & \text { if } i=1, \ldots, i_{0}, \\ \theta^{i \prime} \leqslant \theta^{i}, & \text { if } i=i_{0}+1, \ldots,\left\lfloor\frac{r+1}{2}\right\rfloor .\end{cases}
$$

The following result shows that increasing uncertainty about political preferences not only improves the chances of the majority winning the election but also reduces the expected cost of voting by reducing the incentive to turnout.

Proposition 8. Let $\theta^{\prime}$ and $\theta$ be two symmetric priors that induce respective equilibrium voting probabilities $\phi^{\prime *}$ and $\phi^{*}$. If $\theta^{\prime}$ is a mean-preserving spread of $\theta$, then for any $n$ (finite or infinite),

(a) $\phi^{*} \leqslant \phi^{*}$

(b) $\bar{B}\left(\theta^{\prime}, \lambda, n\right) \geqslant \bar{B}(\theta, \lambda, n)$

(c) $\bar{W}\left(\theta^{\prime}, \lambda, n\right) \geqslant \bar{W}(\theta, \lambda, n)$.

The intuition behind part (a) is obvious. When agents are more uncertain about political preferences, they expect that the election is less likely to be close, which, in turn, makes their votes less pivotal, curbing the incentive to turnout. This reduces the expected social cost. Part (b) is, however, less obvious. To see this, recall that the expected social benefit increases if and only if the probability of winning for the majority increases. However, by encouraging fewer citizens to vote, greater uncertainty adversely affects this probability. What part (b) shows is that the positive direct effect of greater uncertainty outweighs the negative strategic effect. The last part then follows from parts (a) and (b).

\section{Conclusion}

In this paper we have explored the impact of public information about the composition of the electorate on equilibrium voting behavior. Our theoretical findings demonstrate that providing more information of this kind to potential voters harms the democratic process. It biases electoral outcomes toward the alternative preferred by the minority and it leads to higher expected aggregate voting costs.

Although we have employed a standard costly voting model where voters possess intrinsic preferences (i.e., private values) over electoral alternatives and a fixed identical cost of voting, the model can be extended in various ways to lend additional realism and provide guidance for policymakers. In what follows, we briefly discuss three possible extensions of the basic model. 
- Cost uncertainty. Our assumption that all agents possess the same cost of voting is clearly a dramatic simplification. We have, however, investigated a more realistic setting where agents' costs are drawn independently from a continuous distribution $G(\cdot)$ defined on $\left[c_{0}, c_{1}\right]$ as in Palfrey and Rosenthal (1985). Our analysis revealed that all results except for the weak neutrality finding in Proposition 1 remain essentially unchanged. In particular, the strong neutrality result under the informed regime, and the main welfare conclusions continue to hold under cost (or benefit) heterogeneity. The breakdown of weak neutrality under the informed regime implies that the majority is more likely to win the election when the population is finite, although an under-dog effect still obtains that gives the minority a disproportionate probability of winning. The intuition is as follows. As noted in Proposition 1, weak neutrality requires that voting cost not be so small as to violate the mixed strategy equilibrium. Otherwise, even if the minority agents vote with probability 1 , they will be unable to completely neutralize the majority, and thus for small costs, the majority is more likely to win. When costs are drawn from a continuous distribution, the equilibrium must account for all possible cost realizations (including the small ones), implying that the majority wins with probability greater than $\frac{1}{2}$. In a large election, however, only the agents whose costs are close to the lower bound $c_{0}$ vote. In this case, the equilibrium conditions coincide with the setting studied above with voting cost equal to $c_{0}$. Hence, the probability that either side wins is $1 / 2$ independent of $\lambda$ in this case.

- Common values. It seems plain that in many elections voters are motivated primarily by their fundamental ideologies. Party affiliations and political labels such as Liberal and Conservative obviously connote intrinsic differences in ideological principles. Hence, in elections where ideologies are especially important, the findings presented in this paper regarding the release of public information sound a precautionary note. Nonetheless, it is reasonable to suppose that some fraction of citizens may be non partisans whose objective is to elect the "best" or "most qualified" candidate. It would, therefore, be very interesting to investigate the role of public information in a richer model that allows for both partisan and nonpartisan citizens.

- Endogenous source of information. To the extent that opinion polls, political stock markets, and expert forecasts contain real information about voter sentiment, our analysis reveals that their publication may actually impede efficiency, giving rise to closely contested elections with excessive voter turnout. Before firm policy conclusions can be drawn, however, future research must investigate the endogeneity of the source of information about political preferences. In particular, given the feedback we find between information and equilibrium voting behavior, it is important to understand the incentives for individuals to report their true preferences to pollsters and the incentives for pollsters and pundits to disclose publicly and fully any information they obtain. ${ }^{22}$

\section{Appendix A}

Proof of Lemma 1. Suppose $0<\phi_{t}<1$ for $t=A, B$. First, note that a type $t$ agent's respective expected payoffs from voting and abstaining are given by

$$
\begin{aligned}
U_{t}^{1}= & \sum_{k=0}^{n-1}\left(\begin{array}{c}
n-1 \\
k
\end{array}\right) \lambda_{t}^{k}\left(1-\lambda_{t}\right)^{n-1-k} \sum_{k_{t}=0}^{k}\left(\begin{array}{c}
k \\
k_{t}
\end{array}\right) \phi_{t}^{k_{t}}\left(1-\phi_{t}\right)^{k-k_{t}} \\
& \times\left[\sum_{k_{t^{\prime}}=0}^{k_{t}-1}\left(\begin{array}{c}
n-1-k \\
k_{t^{\prime}}
\end{array}\right) \phi_{t^{\prime}}^{k_{t^{\prime}}}\left(1-\phi_{t^{\prime}}\right)^{n-1-k-k_{t^{\prime}}}+\left(\begin{array}{c}
n-1-k \\
k_{t}
\end{array}\right) \phi_{t^{\prime}}^{k_{t}}\left(1-\phi_{t^{\prime}}\right)^{n-1-k-k_{t}}\right. \\
& \left.+\frac{1}{2}\left(\begin{array}{c}
n-1-k \\
k_{t}+1
\end{array}\right) \phi_{t^{\prime}}^{k_{t}+1}\left(1-\phi_{t^{\prime}}\right)^{n-2-k-k_{t}}\right]-c,
\end{aligned}
$$

and

$$
\begin{aligned}
U_{t}^{0}= & \sum_{k=0}^{n-1}\left(\begin{array}{c}
n-1 \\
k
\end{array}\right) \lambda_{t}^{k}\left(1-\lambda_{t}\right)^{n-1-k} \sum_{k_{t}=0}^{k}\left(\begin{array}{c}
k \\
k_{t}
\end{array}\right) \phi_{t}^{k_{t}}\left(1-\phi_{t}\right)^{k-k_{t}} \\
& \times\left[\sum_{k_{t^{\prime}}=0}^{k_{t}-1}\left(\begin{array}{c}
n-1-k \\
k_{t^{\prime}}
\end{array}\right) \phi_{t^{\prime}}^{k_{t^{\prime}}}\left(1-\phi_{t^{\prime}}\right)^{n-1-k-k_{t^{\prime}}}+\frac{1}{2}\left(\begin{array}{c}
n-1-k \\
k_{t}
\end{array}\right) \phi_{t^{\prime}}^{k_{t}}\left(1-\phi_{t^{\prime}}\right)^{n-1-k-k_{t}}\right] .
\end{aligned}
$$

To understand these expected payoffs, fix a type $t$ agent, and let $k_{t}$ be the number of votes for alternative $t$ excluding his, and $k_{t^{\prime}}$ be the number of all votes for alternative $t^{\prime}$. Clearly, if $k_{t^{\prime}} \leqslant k_{t}-1$ and $k_{t^{\prime}} \geqslant k_{t}+2$, then alternative $t$ respectively wins and loses with probability 1 , regardless of the type $t$ agent's action. If $k_{t^{\prime}}=k_{t}$, alternative $t$ wins with probability 1 if the type $t$ agent in question votes, and wins with probability $\frac{1}{2}$ if he abstains and leaves the tie. Finally, if $k_{t^{\prime}}=k_{t}+1$, alternative

\footnotetext{
22 Ottaviani and Sorensen (2006) consider competition among forecasters whose payoffs depend on the ex post accuracy of their forecasts. The difference in an election context is that accuracy depends on the voters' endogenous behavior.
} 
$t$ loses with probability 1 if the type $t$ agent abstains; but may win with probability $\frac{1}{2}$ if he votes. These events explain the expressions in parentheses above. The first two summations in $U_{t}^{1}$ and $U_{t}^{0}$ account for the distribution of preferences.

Next, subtracting $U_{t}^{0}$ from $U_{t}^{1}$, the third summation inside parentheses cancel out, reducing the net expected payoff to

$$
\begin{aligned}
\Delta_{t}=U_{t}^{1}-U_{t}^{0}= & \sum_{k=0}^{n-1}\left(\begin{array}{c}
n-1 \\
k
\end{array}\right) \lambda_{t}^{k}\left(1-\lambda_{t}\right)^{n-1-k} \sum_{k_{t}=0}^{k}\left(\begin{array}{c}
k \\
k_{t}
\end{array}\right) \phi_{t}^{k_{t}}\left(1-\phi_{t}\right)^{k-k_{t}}\left[\frac{1}{2}\left(\begin{array}{c}
n-1-k \\
k_{t}
\end{array}\right) \phi_{t^{\prime}}^{k_{t}}\left(1-\phi_{t^{\prime}}\right)^{n-1-k-k_{t}}\right. \\
& \left.+\frac{1}{2}\left(\begin{array}{c}
n-1-k \\
k_{t}+1
\end{array}\right) \phi_{t^{\prime}}^{k_{t}+1}\left(1-\phi_{t^{\prime}}\right)^{n-2-k-k_{t}}\right]-c .
\end{aligned}
$$

Now, recall $\alpha_{t}=\lambda_{t} \phi_{t}$ and define $\beta_{t}=\lambda_{t}\left(1-\phi_{t}\right)$. By substituting for these terms into (A.1), and noting the following facts,

$$
\begin{aligned}
\lambda_{t}^{k} & =\lambda_{t}^{k_{t}} \lambda_{t}^{k-k_{t}}, \\
\left(1-\lambda_{t}\right)^{n-1-k} & =\left(1-\lambda_{t}\right)^{k_{t}}\left(1-\lambda_{t}\right)^{n-1-k-k_{t},} \\
\left(1-\lambda_{t}\right)^{n-1-k} & =\left(1-\lambda_{t}\right)^{k_{t}+1}\left(1-\lambda_{t}\right)^{n-2-k-k_{t}},
\end{aligned}
$$

Eq. (A.1) further reduces to

$$
\begin{aligned}
\Delta_{t}= & \frac{1}{2} \sum_{k=0}^{n-1}\left[\left(\begin{array}{c}
n-1 \\
k
\end{array}\right) \sum_{k_{t}=0}^{k}\left(\begin{array}{c}
k \\
k_{t}
\end{array}\right) \alpha_{t}^{k_{t}} \beta_{t}^{k-k_{t}}\right. \\
& \left.\times\left(\left(\begin{array}{c}
n-1-k \\
k_{t}
\end{array}\right) \alpha_{t^{\prime}}^{k_{t}} \beta_{t^{\prime}}^{n-1-k-k_{t}}+\left(\begin{array}{c}
n-1-k \\
k_{t}+1
\end{array}\right) \alpha_{t^{\prime}}^{k_{t}+1} \beta_{t^{\prime}}^{n-2-k-k_{t}}\right)\right]-c \\
= & \frac{1}{2} \sum_{k_{t}=0}^{\left\lfloor\frac{n-1}{2}\right\rfloor} \sum_{k=0}^{n-1}\left(\begin{array}{c}
n-1 \\
k
\end{array}\right)\left(\begin{array}{c}
k \\
k_{t}
\end{array}\right)\left(\begin{array}{c}
n-1-k \\
k_{t}
\end{array}\right) \alpha_{t}^{k_{t}} \alpha_{t^{\prime}}^{k_{t}} \beta_{t}^{k-k_{t}} \beta_{t^{\prime}}^{n-1-k-k_{t}} \\
& +\frac{1}{2} \sum_{k_{t}=0}^{\left\lfloor\frac{n-2}{2}\right\rfloor} \sum_{k=0}^{n-1}\left(\begin{array}{c}
n-1 \\
k
\end{array}\right)\left(\begin{array}{c}
k \\
k_{t}
\end{array}\right)\left(\begin{array}{c}
n-1-k \\
k_{t}+1
\end{array}\right) \alpha_{t}^{k_{t}} \alpha_{t^{\prime}}^{k_{t}+1} \beta_{t}^{k-k_{t}} \beta_{t^{\prime}}^{n-2-k-k_{t}}-c .
\end{aligned}
$$

Using the following two combinatorial identities:

$$
\left(\begin{array}{c}
n-1 \\
k
\end{array}\right)\left(\begin{array}{c}
k \\
k_{t}
\end{array}\right)\left(\begin{array}{c}
n-1-k \\
k_{t}
\end{array}\right)=\left(\begin{array}{c}
n-1 \\
k_{t}, k_{t}, n-1-2 k_{t}
\end{array}\right)\left(\begin{array}{c}
n-1-2 k_{t} \\
k-k_{t}
\end{array}\right)
$$

and

$$
\left(\begin{array}{c}
n-1 \\
k
\end{array}\right)\left(\begin{array}{c}
k \\
k_{t}
\end{array}\right)\left(\begin{array}{c}
n-1-k \\
k_{t}+1
\end{array}\right)=\left(\begin{array}{c}
n-1 \\
k_{t}, k_{t}+1, n-2-2 k_{t}
\end{array}\right)\left(\begin{array}{c}
n-2-2 k_{t} \\
k-k_{t}
\end{array}\right),
$$

$\Delta_{t}$ becomes

$$
\begin{aligned}
\Delta_{t}= & \frac{1}{2} \sum_{k_{t}=0}^{\left\lfloor\frac{n-1}{2}\right\rfloor}\left(\begin{array}{c}
n-1 \\
k_{t}, k_{t}, n-1-2 k_{t}
\end{array}\right) \alpha_{t}^{k_{t}} \alpha_{t^{\prime}}^{k_{t}} \sum_{k=0}^{n-1}\left(\begin{array}{c}
n-1-2 k_{t} \\
k-k_{t}
\end{array}\right) \beta_{t}^{k-k_{t}} \beta_{t^{\prime}}^{n-1-k-k_{t}} \\
& +\frac{1}{2} \sum_{k_{t}=0}^{\left\lfloor\frac{n-2}{2}\right\rfloor}\left(\begin{array}{c}
n-1 \\
k_{t}, k_{t}+1, n-2-2 k_{t}
\end{array}\right) \alpha_{t}^{k_{t}} \alpha_{t^{\prime}}^{k_{t}+1} \sum_{k=0}^{n-1}\left(\begin{array}{c}
n-2-2 k_{t} \\
k-k_{t}
\end{array}\right) \beta_{t}^{k-k_{t}} \beta_{t^{\prime}}^{n-2-k-k_{t}}-c \\
= & \frac{1}{2} \sum_{k=0}^{\left\lfloor\frac{n-1}{2}\right\rfloor}\left(\begin{array}{c}
n-1 \\
k, k, n-1-2 k
\end{array}\right) \alpha_{t}^{k} \alpha_{t^{\prime}}^{k}\left(\beta_{t}+\beta_{t^{\prime}}\right)^{n-1-2 k} \\
& +\frac{1}{2} \sum_{k=0}^{\left\lfloor\frac{n-2}{2}\right\rfloor}\left(\begin{array}{c}
n-1 \\
k, k+1, n-2-2 k
\end{array}\right) \alpha_{t}^{k} \alpha_{t^{\prime}}^{k+1}\left(\beta_{t}+\beta_{t^{\prime}}\right)^{n-2-2 k}-c,
\end{aligned}
$$

where we also use the facts that

$$
\sum_{k=0}^{n-1}\left(\begin{array}{c}
n-1-2 k_{t} \\
k-k_{t}
\end{array}\right) \beta_{t}^{k-k_{t}} \beta_{t^{\prime}}^{n-1-k-k_{t}}=\sum_{k=0}^{n-1}\left(\begin{array}{c}
n-1-2 k_{t} \\
k-k_{t}
\end{array}\right) \beta_{t}^{k-k_{t}} \beta_{t^{\prime}}^{n-1-2 k_{t}-\left(k-k_{t}\right)}=\left(\beta_{t}+\beta_{t^{\prime}}\right)^{n-1-2 k_{t}}
$$


and

$$
\sum_{k=0}^{n-1}\left(\begin{array}{c}
n-2-2 k_{t} \\
k-k_{t}
\end{array}\right) \beta_{t}^{k-k_{t}} \beta_{t^{\prime}}^{n-2-k-k_{t}}=\sum_{k=0}^{n-1}\left(\begin{array}{c}
n-2-2 k_{t} \\
k-k_{t}
\end{array}\right) \beta_{t}^{k-k_{t}} \beta_{t^{\prime}}^{n-2-2 k_{t}-\left(k-k_{t}\right)}=\left(\beta_{t}+\beta_{t^{\prime}}\right)^{n-2-2 k_{t}}
$$

and, w.l.o.g., change index of summations to $k$ in the last equality.

The expressions in (1) and (2) then follows by simply observing that $\beta_{A}+\beta_{B}=1-\alpha_{A}-\alpha_{B}$.

Proof of Proposition 1. Suppose $\left(\phi_{A}, \phi_{B}\right)$ is a symmetric BNE in totally mixed strategies, i.e., $0<\phi_{t}<1$ for $t=A, B$. Then, $\Delta_{t}=0$, or equivalently

$$
\frac{1}{2} P_{t}\left(\alpha_{t}, \alpha_{t^{\prime}}\right)-c=0
$$

Solving (A.2) for $\alpha_{t^{\prime}}$, we obtain

$$
\alpha_{t^{\prime}}=\frac{2 c-\sum_{k=0}^{\left\lfloor\frac{n-1}{2}\right\rfloor}\left(\begin{array}{c}
n-1 \\
k, k, n-1-2 k
\end{array}\right) \alpha_{t}^{k} \alpha_{t^{\prime}}^{k}\left(1-\alpha_{t}-\alpha_{t^{\prime}}\right)^{n-1-2 k}}{\sum_{k=0}^{\left.L \frac{n-2}{2}\right\rfloor}\left(\begin{array}{c}
n-1 \\
k, k+1, n-2-2 k
\end{array}\right) \alpha_{t}^{k} \alpha_{t^{\prime}}^{k}\left(1-\alpha_{t}-\alpha_{t^{\prime}}\right)^{n-2-2 k}} .
$$

Hence, $\alpha_{A}=\alpha_{B}$, or equivalently $\lambda_{A} \phi_{A}=\lambda_{B} \phi_{B}$.

To prove part (ii), let $\alpha_{t}=\alpha$. Note that the ex ante probability that a citizen votes is $2 \alpha$. Thus, the probability that alternative $A$ wins given that an odd number $k$ of citizens vote is

$$
\frac{\left(\begin{array}{l}
n \\
k
\end{array}\right)(1-2 \alpha)^{n-k}(2 \alpha)^{k}\left(\sum_{j=0}^{\frac{k-1}{2}}\left(\begin{array}{l}
k \\
j
\end{array}\right) \alpha^{k-j} \alpha^{j}\right)}{\left(\begin{array}{l}
n \\
k
\end{array}\right)(1-2 \alpha)^{n-k}(2 \alpha)^{k}\left(\sum_{j=0}^{k}\left(\begin{array}{l}
k \\
j
\end{array}\right) \alpha^{k-j} \alpha^{j}\right)} .
$$

Factoring $\alpha^{k}$ out of the summations and canceling like terms in the numerator and denominator yields

$$
\frac{\sum_{j=0}^{\frac{k-1}{2}}\left(\begin{array}{l}
k \\
j
\end{array}\right)}{\sum_{j=0}^{k}\left(\begin{array}{l}
k \\
j
\end{array}\right)}=\frac{1}{2}
$$

where we exploit $\left(\begin{array}{l}k \\ j\end{array}\right)=\left(\begin{array}{c}k \\ k-j\end{array}\right)$.

If an even number $k>0$ of citizens vote, then a tie occurs in the event that $j=\frac{k}{2}$ of them vote for alternative $B$, in which case the election is decided by a coin toss. Hence, the probability that alternative $A$ wins is

$$
\frac{\sum_{j=0}^{\frac{k}{2}-1}\left(\begin{array}{l}
k \\
j
\end{array}\right)+\left(\begin{array}{l}
k \\
\frac{k}{2}
\end{array}\right)\left(\frac{1}{2}\right)}{\sum_{j=0}^{k}\left(\begin{array}{l}
k \\
j
\end{array}\right)}=\frac{1}{2} .
$$

Finally, if $k=0$ citizens vote, then $A$ also wins with probability $\frac{1}{2}$.

Proof of Lemma 2. We simply differentiate $P(\alpha, n)$ with respect to $\alpha$

$$
\begin{aligned}
\frac{\partial}{\partial \alpha} P(\alpha, n)= & {\left[\sum_{k=1}^{\left\lfloor\frac{n-1}{2}\right\rfloor} \frac{(n-1) !}{(k !)^{2}(n-1-2 k) !} 2 k \alpha^{2 k-1}(1-2 \alpha)^{n-1-2 k}\right.} \\
& \left.-\sum_{k=0}^{\left\lfloor\frac{n-1}{2}\right\rfloor} \frac{(n-1) !}{(k !)^{2}(n-1-2 k) !} \alpha^{2 k} 2(n-1-2 k)(1-2 \alpha)^{n-2-2 k}\right] \\
& +\left[\sum_{k=0}^{\left\lfloor\frac{n-2}{2}\right\rfloor} \frac{(n-1) !}{k !(k+1) !(n-2-2 k) !}(2 k+1) \alpha^{2 k}(1-2 \alpha)^{n-2-2 k}\right. \\
& \left.-\sum_{k=0}^{\left\lfloor\frac{n-2}{2}\right\rfloor} \frac{(n-1) !}{k !(k+1) !(n-2-2 k) !} \alpha^{2 k} 2(n-2-2 k)(1-2 \alpha)^{n-3-2 k}\right] .
\end{aligned}
$$

Next, we combine the first summation with the last one and the second summation with the third one, 


$$
\begin{aligned}
\frac{\partial}{\partial \alpha} P(\alpha, n) \leqslant & (n-1) !\left[\sum_{k=1}^{\left\lfloor\frac{n-1}{2}\right\rfloor} \frac{2 k}{(k !)^{2}(n-1-2 k) !} \alpha^{2 k-1}(1-2 \alpha)^{n-1-2 k}\right. \\
& \left.-\sum_{k=0}^{\left\lfloor\frac{n-2}{2}\right\rfloor} \frac{2(n-2-2 k)}{k !(k+1) !(n-2-2 k) !} \alpha^{2 k+1}(1-2 \alpha)^{n-3-2 k}\right] \\
& +(n-1) ! \sum_{k=0}^{\left\lfloor\frac{n-2}{2}\right\rfloor}\left(\frac{2 k+1}{k !(k+1) !(n-2-2 k) !}-\frac{2(n-1-2 k)}{(k !)^{2}(n-1-2 k) !}\right) \alpha^{2 k}(1-2 \alpha)^{n-2-2 k} \\
= & (n-1) !\left[\sum_{k=0}^{\left\lfloor\frac{n-1}{2}\right\rfloor-1} \frac{2(k+1)}{((k+1) !)^{2}(n-1-2(k+1)) !} \alpha^{2 k+1}(1-2 \alpha)^{n-3-2 k}\right. \\
& \left.-\sum_{k=0}^{\left\lfloor\frac{n-2}{2}\right\rfloor} \frac{2(n-2-2 k)}{k !(k+1) !(n-2-2 k) !} \alpha^{2 k+1}(1-2 \alpha)^{n-3-2 k}\right] \\
& +(n-1) ! \sum_{k=0}^{\left\lfloor\frac{n-2}{2}\right\rfloor}\left(\frac{2 k+1}{k !(k+1) !(n-2-2 k) !}-\frac{2}{(k !)^{2}(n-2-2 k) !}\right) \alpha^{2 k}(1-2 \alpha)^{n-2-2 k} \\
\leqslant & (n-1) ! \sum_{k=0}^{\left\lfloor\frac{n-1}{2}\right\rfloor-1}\left(\frac{2(n-2-2 k)}{k !(k+1) !(n-2-2 k) !}-\frac{2(k+1)}{((k+1) !)^{2}(n-1-2(k+1)) !}\right) \alpha^{2 k+1}(1-2 \alpha)^{n-3-2 k} \\
& +(n-1) ! \sum_{k=0}^{\left\lfloor\frac{n-2}{2}\right\rfloor}\left(\frac{2 k+1}{k !(k+1) !(n-2-2 k) !}-\frac{2}{(k !)^{2}(n-2-2 k) !}\right) \alpha^{2 k}(1-2 \alpha)^{n-2-2 k} .
\end{aligned}
$$

Because

$$
\frac{2(k+1)}{((k+1) !)^{2}(n-1-2(k+1)) !}-\frac{2(n-2-2 k)}{k !(k+1) !(n-2-2 k) !}=0
$$

and

$$
\frac{2 k+1}{k !(k+1) !(n-2-2 k) !}-\frac{2}{(k !)^{2}(n-2-2 k) !}=-\frac{1}{k !(k+1) !(n-2-2 k) !},
$$

it follows that

$$
\frac{\partial}{\partial \alpha} P(\alpha, n) \leqslant-(n-1) ! \sum_{k=0}^{\left\lfloor\frac{n-2}{2}\right\rfloor} \frac{1}{k !(k+1) !(n-2-2 k) !} \alpha^{2 k}(1-2 \alpha)^{n-2-2 k}<0 .
$$

Proof of Proposition 2. Since, from Lemma 2, $P(\alpha, n)$ is strictly decreasing in $\alpha \in\left(0, \frac{1}{2}\right)$, there is at most one solution to $\frac{1}{2} P(\alpha, n)-c=0$. Moreover, a solution exists if and only if $\frac{1}{2} P(0, n)-c>0$ and $\frac{1}{2} P\left(\frac{1}{2}, n\right)-c<0$. It is easy to verify that $P(0, n)=1$ and $\frac{1}{2} P\left(\frac{1}{2}, n\right) \equiv \underline{c}(n)$ as given in (6). Hence, there is a unique solution $\alpha^{*}(c, n)$ to Eq. (3) if and only if $\underline{c}(n)<c<\frac{1}{2}$. For $\alpha^{*}(c, n)$ to be part of an equilibrium however, it also needs to satisfy: $0<\phi_{t}=\frac{\alpha^{*}(c, n)}{\lambda_{t}}<1$ and $0<\phi_{t^{\prime}}=$ $\frac{\alpha^{*}(c, n)}{1-\lambda_{t}}<1$, or combining the two: $\alpha^{*}(c, n)<\lambda_{t}<1-\alpha^{*}(c, n)$.

Proof of Lemma 3. We first show by induction that

$$
\underline{c}(n)= \begin{cases}\frac{1}{2} \prod_{k=0}^{\frac{n-1}{2}-1} \frac{2 k+1}{2 k+2}, & \text { if } n \text { is odd, } \\ \frac{1}{2} \prod_{k=0}^{\frac{n}{2}-1} \frac{2 k+1}{2 k+2}, & \text { if } n \text { is even. }\end{cases}
$$

Note that $\underline{c}(2)=\underline{c}(3)=\frac{1}{4}$, which is true by (6). Next, let the expression in (A.3) hold true for some arbitrary $n$. By (6), observe that

$$
\underline{c}(n+1)= \begin{cases}\frac{n}{n+1} \underline{c}(n), & \text { if } n \text { is odd } \\ \underline{c}(n), & \text { if } n \text { is even, }\end{cases}
$$


which coincides with the expression obtained from (A.3) for $\underline{c}(n+1)$, completing the induction argument.

Since $\frac{2 k+1}{2 k+2}<1, \underline{c}(n)$ in (A.3) is clearly decreasing. Moreover, $\underline{c}(n) \leqslant b(n)$ for some sequence $b(n) \equiv \prod_{k=0}^{n} \sqrt{\frac{k+1}{k+2}}$. Simple algebra shows $b(n)=\frac{1}{\sqrt{n+2}}$, which converges to 0 . Hence, $\lim _{n \rightarrow \infty} \underline{c}(n)=0$.

To prove that $\alpha^{*}(c, n)$ is decreasing in $n$, it suffices to prove $P(\alpha, n)$ is decreasing in $n$. By definition,

$$
\begin{aligned}
P(\alpha, n)-P(\alpha, n+1) & \\
= & \sum_{k=0}^{\left\lfloor\frac{n-1}{2}\right\rfloor} \frac{(n-1) !}{(k !)^{2}(n-1-2 k) !} \alpha^{2 k}(1-2 \alpha)^{n-1-2 k}+\sum_{k=0}^{\left\lfloor\frac{n-2}{2}\right\rfloor} \frac{(n-1) !}{k !(k+1) !(n-2-2 k) !} \alpha^{2 k+1}(1-2 \alpha)^{n-2-2 k} \\
& -\sum_{k=0}^{\left\lfloor\frac{n}{2}\right\rfloor} \frac{n !}{(k !)^{2}(n-2 k) !} \alpha^{2 k}(1-2 \alpha)^{n-2 k}-\sum_{k=0}^{\left\lfloor\frac{n-1}{2}\right\rfloor} \frac{n !}{k !(k+1) !(n-1-2 k) !} \alpha^{2 k+1}(1-2 \alpha)^{n-1-2 k} .
\end{aligned}
$$

Before signing this expression, we suppose that $n$ is odd, and re-write the third summation:

$$
\begin{aligned}
& \sum_{k=0}^{\left\lfloor\frac{n}{2}\right\rfloor} \frac{n !}{(k !)^{2}(n-2 k) !} \alpha^{2 k}(1-2 \alpha)^{n-2 k} \\
& \quad=\sum_{k=0}^{\frac{n-1}{2}}\left[1+\frac{2 k}{n-2 k}\right] \frac{(n-1) !}{(k !)^{2}(n-1-2 k) !} \alpha^{2 k}(1-2 \alpha)^{n-2 k} \\
& \quad=(1-2 \alpha) \sum_{k=0}^{\frac{n-1}{2}} \frac{(n-1) !}{(k !)^{2}(n-1-2 k) !} \alpha^{2 k}(1-2 \alpha)^{n-1-2 k}+2 \alpha \sum_{k=1}^{\frac{n-1}{2}} \frac{(n-1) !}{(k-1) ! k !(n-2 k) !} \alpha^{2 k-1}(1-2 \alpha)^{n-2 k} \\
& \quad=(1-2 \alpha) \sum_{k=0}^{\frac{n-1}{2}} \frac{(n-1) !}{(k !)^{2}(n-1-2 k) !} \alpha^{2 k}(1-2 \alpha)^{n-1-2 k}+2 \alpha \sum_{k=0}^{\frac{n-1}{2}-1} \frac{(n-1) !}{k !(k+1) !(n-2-2 k) !} \alpha^{2 k+1}(1-2 \alpha)^{n-2-2 k}
\end{aligned}
$$

Inserting this into (A.4) and canceling terms yield

$$
\begin{aligned}
& P(\alpha, n)-P(\alpha, n+1)=2 \alpha \sum_{k=0}^{\frac{n-1}{2}} \frac{(n-1) !}{(k !)^{2}(n-1-2 k) !} \alpha^{2 k}(1-2 \alpha)^{n-1-2 k} \\
& +(1-2 \alpha) \sum_{k=0}^{\frac{n-1}{2}-1} \frac{(n-1) !}{k !(k+1) !(n-2-2 k) !} \alpha^{2 k+1}(1-2 \alpha)^{n-2-2 k} \\
& -\sum_{k=0}^{\frac{n-1}{2}} \frac{n !}{k !(k+1) !(n-1-2 k) !} \alpha^{2 k+1}(1-2 \alpha)^{n-1-2 k} \\
& =\sum_{k=0}^{\frac{n-1}{2}-1}\left(\frac{2}{n-1-2 k}+\frac{1}{k+1}\right) \frac{(n-1) !}{(k !)^{2}(n-2-2 k) !} \alpha^{2 k+1}(1-2 \alpha)^{n-1-2 k} \\
& -\sum_{k=0}^{\frac{n-1}{2}} \frac{n !}{k !(k+1) !(n-1-2 k) !} \alpha^{2 k+1}(1-2 \alpha)^{n-1-2 k}+\frac{2(n-1) !}{\left(\frac{n-1}{2} !\right)^{2}} \alpha^{n} \\
& =\sum_{k=0}^{\frac{n-1}{2}-1}((n+1)-n) \frac{(n-1) !}{k !(k+1) !(n-1-2 k) !} \alpha^{2 k+1}(1-2 \alpha)^{n-1-2 k} \\
& +\frac{2(n-1) !}{\left(\frac{n-1}{2} !\right)^{2}} \alpha^{n}-\frac{n !}{\frac{n-1}{2} !\left(\frac{n-1}{2}+1\right) !} \alpha^{n}>0 .
\end{aligned}
$$

Given that $\alpha^{*}(c, n)$ is decreasing in $n$ and bounded below by 0 , it converges to some $\alpha_{\ell} \geqslant 0$. Suppose $\alpha_{\ell}>0$. Since $P(\alpha, n)$ is continuous in $\alpha$, it follows that $\lim _{n \rightarrow \infty} \frac{1}{2} P\left(\alpha_{\ell}, n\right)-c=0$, or $\lim _{n \rightarrow \infty} P\left(\alpha_{\ell}, n\right)=2 c$. 
We now argue that if $\alpha_{\ell}>0$, then $\lim _{n \rightarrow \infty} P\left(\alpha_{\ell}, n\right)=0$. To see this, let $X_{t}$ be the number of votes for alternative $t$ (as in the text), and $Y=X_{A}-X_{B}$ be the vote difference. Then,

$$
P\left(\alpha_{\ell}, n\right)=\operatorname{Pr}\{Y=0\}+\operatorname{Pr}\{Y=1\} .
$$

For a large $n$, we have $Y \stackrel{D}{\rightarrow} \operatorname{Normal}\left(0,2 n \alpha_{\ell}\right)$. Hence, as $n \rightarrow \infty$, it follows that $\operatorname{Pr}\{Y=0\} \rightarrow 0$ and $\operatorname{Pr}\{Y=1\} \rightarrow 0$, implying that $P\left(\alpha_{\ell}, n\right) \rightarrow 0$. But this contradicts $\lim _{n \rightarrow \infty} P\left(\alpha_{\ell}, n\right)=2 c \neq 0$. Hence, $\alpha_{\ell}=0$. The same exact arguments hold for an even $n$.

The last part simply follows from (3), which holds for any $n$ in a totally mixed strategy equilibrium.

Proof of Lemma 4. Note that $\left(X_{A} \mid X_{B}\right) \sim \operatorname{Binomial}\left(n-X_{B}, \frac{\alpha_{A}^{*}(n)}{1-\alpha_{B}^{*}(n)}\right)$, where $\frac{\alpha_{A}^{*}(n)}{1-\alpha_{B}^{*}(n)} \in(0,1)$ since $0<\alpha_{t}^{*}(n)<\lambda_{t}($ and thus $\left.0<\alpha_{A}^{*}(n)+\alpha_{B}^{*}(n)<1\right)$. Moreover, for a fixed $X_{B}$, since $\lim _{n \rightarrow \infty} \alpha_{t}^{*}(n)=0$ by Lemma 3 , it follows

$$
\lim _{n \rightarrow \infty}\left[\left(n-X_{B}\right) \frac{\alpha_{A}^{*}(n)}{1-\alpha_{B}^{*}(n)}\right]=m_{A} .
$$

Hence (see Billingsley, 1995, Theorem 23.2)

$$
\left(X_{A} \mid X_{B}\right) \stackrel{D}{\rightarrow} \text { Poisson }\left(m_{A}\right)
$$

which is independent of $X_{B}$. The same argument shows

$$
\left(X_{B} \mid X_{A}\right) \stackrel{D}{\rightarrow} \operatorname{Poisson}\left(m_{B}\right) \text {. }
$$

Hence, the limiting distributions of $X_{A}$ and $X_{B}$ are independent, which further implies

$$
\left(X_{A}+X_{B}\right) \stackrel{D}{\rightarrow} \operatorname{Poisson}\left(m_{A}+m_{B}\right) .
$$

Proof of Proposition 3. Fix any pair of $\left(\lambda_{t}, c\right) \in(0,1) \times\left(0, \frac{1}{2}\right)$. By Proposition 2 , there is a unique symmetric mixed-strategy BNE if and only if $c \in\left(\underline{c}(n), \frac{1}{2}\right)$ and $\lambda_{t} \in\left(\alpha^{*}(c, n), 1-\alpha^{*}(c, n)\right)$. Since as $n$ grows, both $\underline{c}(n)$ and $\alpha^{*}(c, n)$ decrease and converge to 0 by Lemma 3, there exist two thresholds $n^{\prime}(c), n^{\prime \prime}\left(\lambda_{t}\right)<\infty$ such that $c \in\left(\underline{c}(n), \frac{1}{2}\right)$ for all $n>n^{\prime}(c)$ and $\lambda_{t} \in$ $\left(\alpha^{*}(c, n), 1-\alpha^{*}(c, n)\right)$ for all $n>n^{\prime \prime}\left(\lambda_{t}\right)$. Define $\bar{n}=\max \left\{n^{\prime}(c), n^{\prime \prime}\left(\lambda_{t}\right)\right\}$. Then, there exists a unique symmetric BNE for all $n \geqslant \bar{n}$.

Part (a) follows from Proposition 1 and Lemma 3. To prove part (b), first note that Eq. (9) implies $m_{A}^{I}=m_{B}^{I}$. This means, after substituting for $m_{A}^{I}=m_{B}^{I}=\frac{M}{2}$, Eq. (9) reduces to $\Phi^{I}\left(\frac{M}{2}, \frac{M}{2}\right)=2 c$, where $\Phi^{I}\left(\frac{M}{2}, \frac{M}{2}\right)=\sum_{k=0}^{\infty} \frac{\left(\frac{M}{2}\right)^{2 k} e^{-M}}{(k !)^{2}}+$ $\sum_{k=0}^{\infty} \frac{\left(\frac{M}{2}\right)^{2 k+1} e^{-M}}{k !(k+1) !}$ by (8). Next, we show that $\Phi^{I}\left(\frac{M}{2}, \frac{M}{2}\right)$ is strictly decreasing in $M$. Differentiation yields

$$
\begin{aligned}
& \frac{d}{d M} \Phi^{I}\left(\frac{M}{2}, \frac{M}{2}\right)=e^{-M}\left[\sum_{k=1}^{\infty} \frac{1}{2^{2 k}} \frac{2 k M^{2 k-1}}{(k !)^{2}}+2 \sum_{k=0}^{\infty} \frac{1}{2^{2(k+1)}} \frac{(2 k+1) M^{2 k}}{k !(k+1) !}\right. \\
& \left.-\sum_{k=0}^{\infty} \frac{1}{2^{2 k}} \frac{M^{2 k}}{(k !)^{2}}-2 \sum_{k=0}^{\infty} \frac{1}{2^{2(k+1)}} \frac{M^{2 k+1}}{k !(k+1) !}\right] \\
& =e^{-M}\left[\sum_{k=0}^{\infty} \frac{1}{2^{2(k+1)}} \frac{2(k+1) M^{2(k+1)-1}}{((k+1) !)^{2}}+2 \sum_{k=0}^{\infty} \frac{1}{2^{2(k+1)}} \frac{(2 k+1) M^{2 k}}{k !(k+1) !}\right. \\
& \left.-\sum_{k=0}^{\infty} \frac{1}{2^{2 k}} \frac{M^{2 k}}{(k !)^{2}}-2 \sum_{k=0}^{\infty} \frac{1}{2^{2(k+1)}} \frac{M^{2 k+1}}{k !(k+1) !}\right] \text {. }
\end{aligned}
$$

Since the first and last summations cancel out, we have

$$
\begin{aligned}
\frac{d}{d M} \Phi^{I}\left(\frac{M}{2}, \frac{M}{2}\right) & =e^{-M}\left[2 \sum_{k=0}^{\infty} \frac{1}{2^{2(k+1)}} \frac{(2 k+1) M^{2 k}}{k !(k+1) !}-\sum_{k=0}^{\infty} \frac{1}{2^{2 k}} \frac{M^{2 k}}{(k !)^{2}}\right] \\
& =e^{-M} \sum_{k=0}^{\infty}\left[\frac{\frac{1}{2}(2 k+1)}{k+1}-1\right] \frac{1}{2^{2 k}} \frac{M^{2 k}}{(k !)^{2}}<0 .
\end{aligned}
$$

It is easy to verify that $\Phi^{I}(0,0)=1$ and $\lim _{M \rightarrow \infty} \Phi^{I}\left(\frac{M}{2}, \frac{M}{2}\right)=0$, which imply

$$
\Phi^{I}(0,0)-2 \underline{c}>0 \text { and } \lim _{M \rightarrow \infty}\left[\Phi^{I}\left(\frac{M}{2}, \frac{M}{2}\right)-2 \underline{c}\right]<0 \text {. }
$$

Hence, there is a unique $M^{I} \in(0, \infty)$ that satisfies $\Phi^{I}\left(\frac{M}{2}, \frac{M}{2}\right)=2 \underline{c}$. 
Proof of Proposition 4. To prove part (a), suppose $\left(\phi_{A}, \phi_{B}\right)$ is a symmetric BNE in totally mixed strategies. Then, Eq. (10) implies

$$
E\left[P_{A}\left(\lambda_{A} \phi_{A},\left(1-\lambda_{A}\right) \phi_{B}\right) \mid A\right]=E\left[P_{B}\left(\left(1-\lambda_{A}\right) \phi_{B}, \lambda_{A} \phi_{A}\right) \mid B\right] .
$$

Next, we establish

$$
\left.E\left[P_{B}\left(1-\lambda_{A}\right) \phi_{B}, \lambda_{A} \phi_{A}\right) \mid B\right]=E\left[P_{A}\left(\lambda_{A} \phi_{B},\left(1-\lambda_{A}\right) \phi_{A}\right) \mid A\right] .
$$

To see this, note from (2) that

$$
\begin{aligned}
E & {\left[P_{B}\left(\left(1-\lambda_{A}\right) \phi_{B}, \lambda_{A} \phi_{A}\right) \mid B\right] } \\
= & \sum_{k=0}^{\left\lfloor\frac{n-1}{2}\right\rfloor}\left[\left(\begin{array}{c}
n-1 \\
k, k, n-1-2 k
\end{array}\right) \sum_{i=1}^{r} 2 \theta^{i}\left(1-\lambda^{i}\right)\left(\lambda^{i} \phi_{A}\right)^{k}\left(\left(1-\lambda^{i}\right) \phi_{B}\right)^{k}\left(1-\lambda^{i} \phi_{A}-\left(1-\lambda^{i}\right) \phi_{B}\right)^{n-1-2 k}\right] \\
& +\sum_{k=0}^{\left\lfloor\frac{n-2}{2}\right\rfloor}\left[\left(\begin{array}{c}
n-1 \\
k, k+1, n-2-2 k
\end{array}\right) \sum_{i=1}^{r} 2 \theta^{i}\left(1-\lambda^{i}\right)\left(\lambda^{i} \phi_{A}\right)^{k+1}\left(\left(1-\lambda^{i}\right) \phi_{B}\right)^{k}\left(1-\lambda^{i} \phi_{A}-\left(1-\lambda^{i}\right) \phi_{B}\right)^{n-2-2 k}\right] .
\end{aligned}
$$

Now, by symmetry of the prior

$$
\begin{aligned}
& \sum_{i=1}^{r} 2 \theta^{i}\left(1-\lambda^{i}\right)\left(\lambda^{i} \phi_{A}\right)^{k}\left(\left(1-\lambda^{i}\right) \phi_{B}\right)^{k}\left(1-\lambda^{i} \phi_{A}-\left(1-\lambda^{i}\right) \phi_{B}\right)^{n-1-2 k} \\
& \quad=\sum_{i=1}^{r}\left[2 \theta^{i} \lambda^{r+1-i}\left(\lambda^{r+1-i} \phi_{B}\right)^{k}\left(\left(1-\lambda^{r+1-i}\right) \phi_{A}\right)^{k}\left(1-\lambda^{r+1-i} \phi_{B}-\left(1-\lambda^{r+1-i}\right) \phi_{A}\right)^{n-1-2 k}\right]
\end{aligned}
$$

and

$$
\begin{aligned}
& \sum_{i=1}^{r} 2 \theta^{i}\left(1-\lambda^{i}\right)\left(\lambda^{i} \phi_{A}\right)^{k+1}\left(\left(1-\lambda^{i}\right) \phi_{B}\right)^{k}\left(1-\lambda^{i} \phi_{A}-\left(1-\lambda^{i}\right) \phi_{B}\right)^{n-2-2 k} \\
& \quad=\sum_{i=1}^{r}\left[2 \theta^{i} \lambda^{r+1-i}\left(\lambda^{r+1-i} \phi_{B}\right)^{k}\left(\left(1-\lambda^{r+1-i}\right) \phi_{A}\right)^{k+1}\left(1-\lambda^{r+1-i} \phi_{B}-\left(1-\lambda^{r+1-i}\right) \phi_{A}\right)^{n-2-2 k}\right] .
\end{aligned}
$$

Substituting these equalities into the above expression for $E\left[P_{B}\left(\left(1-\lambda_{A}\right) \phi_{B}, \lambda_{A} \phi_{A}\right) \mid B\right]$ yields (A.6). Next, combine (A.5) and (A.6) to get

$$
E\left[P_{A}\left(\lambda_{A} \phi_{A},\left(1-\lambda_{A}\right) \phi_{B}\right) \mid A\right]=E\left[P_{A}\left(\lambda_{A} \phi_{B},\left(1-\lambda_{A}\right) \phi_{A}\right) \mid A\right] .
$$

To derive a contradiction to this, suppose w.l.o.g. that $\phi_{A}>\phi_{B}$. We show that this implies

$$
E\left[P_{A}\left(\lambda_{A} \phi_{A},\left(1-\lambda_{A}\right) \phi_{B}\right) \mid A\right]-E\left[P_{A}\left(\lambda_{A} \phi_{B},\left(1-\lambda_{A}\right) \phi_{A}\right) \mid A\right]<0 .
$$

From (2) we have

$$
\begin{aligned}
E[ & \left.P_{A}\left(\lambda_{A} \phi_{A},\left(1-\lambda_{A}\right) \phi_{B}\right) \mid A\right]-E\left[P_{A}\left(\lambda_{A} \phi_{B},\left(1-\lambda_{A}\right) \phi_{A}\right) \mid A\right] \\
= & \sum_{k=0}^{\left\lfloor\frac{n-1}{2}\right\rfloor}\left[\left(\begin{array}{c}
n-1 \\
k, k, n-1-2 k
\end{array}\right) \sum_{i=1}^{r} 2 \theta^{i} \lambda^{i}\left(\lambda^{i}\left(1-\lambda^{i}\right) \phi_{A} \phi_{B}\right)^{k}\right. \\
& \left.\times\left(\left(1-\lambda^{i} \phi_{A}-\left(1-\lambda^{i}\right) \phi_{B}\right)^{n-1-2 k}-\left(1-\lambda^{i} \phi_{B}-\left(1-\lambda^{i}\right) \phi_{A}\right)^{n-1-2 k}\right)\right] \\
& +\sum_{k=0}^{\left\lfloor\frac{n-2}{2}\right\rfloor}\left[\left(\begin{array}{c}
n-1 \\
k, k+1, n-2-2 k
\end{array}\right) \sum_{i=1}^{r} 2 \theta^{i} \lambda^{i}\left(\lambda^{i}\left(1-\lambda^{i}\right) \phi_{A} \phi_{B}\right)^{k}\right. \\
& \left.\times\left(\left(1-\lambda^{i}\right) \phi_{B}\left(1-\lambda^{i} \phi_{A}-\left(1-\lambda^{i}\right) \phi_{B}\right)^{n-2-2 k}-\left(1-\lambda^{i}\right) \phi_{A}\left(1-\lambda^{i} \phi_{B}-\left(1-\lambda^{i}\right) \phi_{A}\right)^{n-2-2 k}\right)\right] .
\end{aligned}
$$

Both of the double summations in this expression are negative. To see that the first one is negative, consider the inner summation 


$$
\sum_{i=1}^{r} 2 \theta^{i} \lambda^{i}\left(\lambda^{i}\left(1-\lambda^{i}\right) \phi_{A} \phi_{B}\right)^{k}\left(\left(1-\lambda^{i} \phi_{A}-\left(1-\lambda^{i}\right) \phi_{B}\right)^{n-1-2 k}-\left(1-\lambda^{i} \phi_{B}-\left(1-\lambda^{i}\right) \phi_{A}\right)^{n-1-2 k}\right) .
$$

Use symmetry of the prior to combine terms 1 and $r, 2$ and $r-1$, and so on, to get

$$
\sum_{i=1}^{\left\lfloor\frac{r+1}{2}\right\rfloor} 2 \theta^{i}\left(2 \lambda^{i}-1\right)\left(\lambda^{i}\left(1-\lambda^{i}\right) \phi_{A} \phi_{B}\right)^{k}\left(\left(1-\lambda^{i} \phi_{A}-\left(1-\lambda^{i}\right) \phi_{B}\right)^{n-1-2 k}-\left(1-\lambda^{i} \phi_{B}-\left(1-\lambda^{i}\right) \phi_{A}\right)^{n-1-2 k}\right) .
$$

(If $r$ is odd, the expression is still valid in this case since the term involving $\lambda_{\left\lfloor\frac{r+1}{2}\right\rfloor}=\frac{1}{2}$ is automatically zero.) If $k=\frac{n-1}{2}$, then each term in this summation is evidently zero. If $k<\frac{n-1}{2}$, then each term is negative. To see this, note that $2 \lambda^{i}-1<0$ because we are summing over the left half of the distribution. Moreover,

$$
\begin{aligned}
\phi_{A}>\phi_{B} & \Rightarrow\left(1-2 \lambda^{i}\right) \phi_{A}>\left(1-2 \lambda^{i}\right) \phi_{B} \\
& \Rightarrow 1-\lambda^{i} \phi_{A}-\left(1-\lambda^{i}\right) \phi_{B}>1-\lambda^{i} \phi_{B}-\left(1-\lambda^{i}\right) \phi_{A} \\
& \Rightarrow\left(1-\lambda^{i} \phi_{A}-\left(1-\lambda^{i}\right) \phi_{B}\right)^{n-1-2 k}>\left(1-\lambda^{i} \phi_{B}-\left(1-\lambda^{i}\right) \phi_{A}\right)^{n-1-2 k} .
\end{aligned}
$$

A similar argument shows that the second double summation in the above expression is also negative.

The proof of part (b) closely follows the proof of its counterpart in Proposition 1. Since, by part (a), each citizen votes with the same probability, $\phi$, the probability that $k$ citizens will turn out to vote is $\left(\begin{array}{l}n \\ k\end{array}\right)(1-\phi)^{n-k} \phi^{k}$. Moreover, since a citizen votes for alternative $t$ with probability $\lambda_{t} \phi$, the probability that alternative $A$ wins when an odd number $k$ of citizens vote is

$$
\frac{\left(\begin{array}{l}
n \\
k
\end{array}\right)(1-\phi)^{n-k} \phi^{k}\left(\sum_{j=0}^{\frac{k-1}{2}}\left(\begin{array}{c}
k \\
j
\end{array}\right)\left(\lambda_{A} \phi\right)^{k-j}\left(\left(1-\lambda_{A}\right) \phi\right)^{j}\right)}{\left(\begin{array}{l}
n \\
k
\end{array}\right)(1-\phi)^{n-k} \phi^{k}\left(\sum_{j=0}^{k}\left(\begin{array}{l}
k \\
j
\end{array}\right)\left(\lambda_{A} \phi\right)^{k-j}\left(\left(1-\lambda_{A}\right) \phi\right)^{j}\right)} .
$$

Factoring $\phi^{k}$ out of the summations and cancelling like terms in the numerator and denominator yields

$$
\frac{\sum_{j=0}^{\frac{k-1}{2}}\left(\begin{array}{l}
k \\
j
\end{array}\right) \lambda_{A}^{k-j}\left(1-\lambda_{A}\right)^{j}}{\sum_{j=0}^{k}\left(\begin{array}{c}
k \\
j
\end{array}\right) \lambda_{A}^{k-j}\left(1-\lambda_{A}\right)^{j}}
$$

Since $\sum_{j=0}^{k}\left(\begin{array}{c}k \\ j\end{array}\right) \lambda_{A}^{k-j}\left(1-\lambda_{A}\right)^{j}=1$, the probability that $A$ wins is equal to

$$
\sum_{j=0}^{\frac{k-1}{2}}\left(\begin{array}{l}
k \\
j
\end{array}\right) \lambda_{A}^{k-j}\left(1-\lambda_{A}\right)^{j}
$$

which is greater than $\frac{1}{2}$ if and only if $\lambda_{A}>\frac{1}{2}$.

If an even number $k>0$ of citizens vote, then a tie occurs in the event that $j=\frac{k}{2}$ of them vote for alternative $B$, in which case the election is decided by a coin toss. Hence, the probability that alternative $A$ wins is

$$
\sum_{j=0}^{\frac{k}{2}-1}\left(\begin{array}{l}
k \\
j
\end{array}\right) \lambda_{A}^{k-j}\left(1-\lambda_{A}\right)^{j}+\left(\begin{array}{l}
k \\
\frac{k}{2}
\end{array}\right) \lambda_{A}^{\frac{k}{2}}\left(1-\lambda_{A}\right)^{\frac{k}{2}}\left(\frac{1}{2}\right)
$$

To evaluate this probability, note that

$$
\begin{aligned}
\sum_{j=0}^{k}\left(\begin{array}{c}
k \\
j
\end{array}\right) \lambda_{A}^{k-j}\left(1-\lambda_{A}\right)^{j}= & \sum_{j=0}^{\frac{k}{2}-1}\left[\left(\begin{array}{c}
k \\
j
\end{array}\right) \lambda_{A}^{k-j}\left(1-\lambda_{A}\right)^{j}+\left(\begin{array}{c}
k \\
\frac{k}{2}
\end{array}\right) \lambda_{A}^{\frac{k}{2}}\left(1-\lambda_{A}\right)^{\frac{k}{2}}\left(\frac{1}{2}\right)\right] \\
& +\sum_{j=\frac{k}{2}+1}^{k}\left[\left(\begin{array}{c}
k \\
j
\end{array}\right) \lambda_{A}^{k-j}\left(1-\lambda_{A}\right)^{j}+\left(\begin{array}{c}
k \\
\frac{k}{2}
\end{array}\right) \lambda_{A}^{\frac{k}{2}}\left(1-\lambda_{A}\right)^{\frac{k}{2}}\left(\frac{1}{2}\right)\right]=1 .
\end{aligned}
$$

Since $\left(\begin{array}{c}k \\ j\end{array}\right)=\left(\begin{array}{c}k \\ k-j\end{array}\right)$, the probability that $A$ wins is strictly greater than $\frac{1}{2}$ if and only if $\lambda_{A}>\frac{1}{2}$.

Finally, if $k=0$ citizens vote, then $A$ wins with probability $\frac{1}{2}$. 
Proof of Proposition 5. Recall from (11)

$$
Q(\phi, n)=\sum_{i=1}^{\left\lfloor\frac{r+1}{2}\right\rfloor} 2 \theta^{i} T\left(\phi, n \mid \lambda_{A}=\lambda^{i}\right),
$$

where $T\left(\phi, n \mid \lambda_{A}=\lambda^{i}\right)$ is defined in (12). First, we show that $Q(\phi, n)$ is decreasing in $\phi$ for $\phi \in(0,1)$. To do so, it suffices to show each term $T\left(\phi, n \mid \lambda_{A}=\lambda^{i}\right)$ is decreasing in $\phi$. Differentiating yields

$$
\begin{aligned}
\frac{\partial}{\partial \phi} T\left(\phi, n \mid \lambda_{A}=\lambda^{i}\right)= & 2 \sum_{k=1}^{\left\lfloor\frac{n-1}{2}\right\rfloor} \frac{(n-1) !}{(k-1) ! k !(n-1-2 k) !}\left(\lambda^{i}\left(1-\lambda^{i}\right)\right)^{k} \phi^{2 k-1}(1-\phi)^{n-1-2 k} \\
& -\sum_{k=0}^{\left\lfloor\frac{n-2}{2}\right\rfloor} \frac{(n-1) !}{(k !)^{2}(n-2-2 k) !}\left(\lambda^{i}\left(1-\lambda^{i}\right)\right)^{k} \phi^{2 k}(1-\phi)^{n-2-2 k} \\
& +2 \lambda^{i}\left(1-\lambda^{i}\right) \sum_{k=0}^{\left\lfloor\frac{n-2}{2}\right\rfloor} \frac{(2 k+1)(n-1) !}{k !(k+1) !(n-2-2 k) !}\left(\lambda^{i}\left(1-\lambda^{i}\right)\right)^{k} \phi^{2 k}(1-\phi)^{n-2-2 k} \\
& -2 \lambda^{i}\left(1-\lambda^{i}\right) \sum_{k=0}^{\left\lfloor\frac{n-3}{2}\right\rfloor} \frac{(n-1) !}{k !(k+1) !(n-3-2 k) !}\left(\lambda^{i}\left(1-\lambda^{i}\right)\right)^{k} \phi^{2 k+1}(1-\phi)^{n-3-2 k} .
\end{aligned}
$$

Since

$$
\begin{aligned}
& 2 \sum_{k=1}^{\left\lfloor\frac{n-1}{2}\right\rfloor} \frac{(n-1) !}{(k-1) ! k !(n-1-2 k) !}\left(\lambda^{i}\left(1-\lambda^{i}\right)\right)^{k} \phi^{2 k-1}(1-\phi)^{n-1-2 k} \\
& \quad=2 \lambda^{i}\left(1-\lambda^{i}\right) \sum_{k=0}^{\left\lfloor\frac{n-1}{2}\right\rfloor-1} \frac{(n-1) !}{(k) !(k+1) !(n-3-2 k) !}\left(\lambda^{i}\left(1-\lambda^{i}\right)\right)^{k} \phi^{2 k+1}(1-\phi)^{n-3-2 k},
\end{aligned}
$$

we have

$$
\begin{aligned}
\frac{\partial}{\partial \phi} T\left(\phi, n \mid \lambda_{A}=\lambda^{i}\right)= & -\sum_{k=0}^{\left\lfloor\frac{n-2}{2}\right\rfloor} \frac{(n-1) !}{(k !)^{2}(n-2-2 k) !}\left(\lambda^{i}\left(1-\lambda^{i}\right)\right)^{k} \phi^{2 k}(1-\phi)^{n-2-2 k} \\
& +2 \lambda^{i}\left(1-\lambda^{i}\right) \sum_{k=0}^{\left\lfloor\frac{n-2}{2}\right\rfloor} \frac{(2 k+1)(n-1) !}{k !(k+1) !(n-2-2 k) !}\left(\lambda^{i}\left(1-\lambda^{i}\right)\right)^{k} \phi^{2 k}(1-\phi)^{n-2-2 k} \\
\leqslant & -\sum_{k=0}^{\left\lfloor\frac{n-2}{2}\right\rfloor} \frac{(n-1) !}{(k !)^{2}(n-2-2 k) !}\left(\lambda^{i}\left(1-\lambda^{i}\right)\right)^{k} \phi^{2 k}(1-\phi)^{n-2-2 k} \\
& +\frac{1}{2} \sum_{k=0}^{\left\lfloor\frac{n-2}{2}\right\rfloor} \frac{(2 k+1)(n-1) !}{k !(k+1) !(n-2-2 k) !}\left(\lambda^{i}\left(1-\lambda^{i}\right)\right)^{k} \phi^{2 k}(1-\phi)^{n-2-2 k} \\
= & -\frac{1}{2} \sum_{k=0}^{\left\lfloor\frac{n-2}{2}\right\rfloor} \frac{(n-1) !}{k !(k+1) !(n-2-2 k) !}\left(\lambda^{i}\left(1-\lambda^{i}\right)\right)^{k} \phi^{2 k}(1-\phi)^{n-2-2 k}<0,
\end{aligned}
$$

where we use the fact that $2 \lambda^{i}\left(1-\lambda^{i}\right) \leqslant \frac{1}{2}$.

Given that $Q(\phi, n)$ is decreasing in $\phi \in(0,1),(13)$ has a unique solution $\phi^{*}(n) \in(0,1)$ if and only if $\frac{1}{2} Q(0, n)-c>0$ and $\frac{1}{2} Q(1, n)-c \leqslant 0$. Noting $Q(0, n)=1$ and defining $\underline{\underline{c}}(n)=\frac{1}{2} Q(1, n)$, the result follows.

Proof of Lemma 5. When $n$ is odd, note that

$$
\underline{\underline{c}}(n)=\underline{c}(n) 2^{n} \sum_{i=1}^{r} \theta^{i}\left(\lambda^{i}\right)^{\frac{n+1}{2}}\left(1-\lambda^{i}\right)^{\frac{n-1}{2}}<\underline{c}(n) 2^{n} \sum_{i=1}^{r} \theta^{i} \lambda^{i}\left(\frac{1}{4}\right)^{\frac{n-1}{2}}
$$




$$
=\underline{c}(n) 2^{n} \frac{1}{2}\left(\frac{1}{4}\right)^{\frac{n-1}{2}}=\underline{c}(n) .
$$

The same argument proves the case for $n$ even.

Proof of Lemma 6. Since $Q(\phi, n)$ is decreasing in $\phi$, it suffices to show $Q(\phi, n)$ is decreasing in $n$ for a fixed $\phi \in(0,1)$. To do so, we prove that each term $T\left(\phi, n \mid \lambda_{A}=\lambda^{i}\right)$ is decreasing in $n$. But this just mimics the proof of the similar result for $\alpha^{*}(n)$ in part (ii) of Lemma 3. In particular,

$$
\begin{aligned}
T\left(\phi, n \mid \lambda_{A}=\lambda^{i}\right)-T\left(\phi, n+1 \mid \lambda_{A}=\lambda^{i}\right)= & \sum_{k=0}^{\left\lfloor\frac{n-1}{2}\right\rfloor} \frac{(n-1) !}{(k !)^{2}(n-1-2 k) !}\left(\lambda^{i}\left(1-\lambda^{i}\right)\right)^{k} \phi^{2 k}(1-\phi)^{n-1-2 k} \\
& +2 \sum_{k=0}^{\left\lfloor\frac{n-2}{2}\right\rfloor} \frac{(n-1) !}{k !(k+1) !(n-2-2 k) !}\left(\lambda^{i}\left(1-\lambda^{i}\right)\right)^{k+1} \phi^{2 k+1}(1-\phi)^{n-2-2 k} \\
& -\sum_{k=0}^{\left\lfloor\frac{n}{2}\right\rfloor} \frac{n !}{(k !)^{2}(n-2 k) !}\left(\lambda^{i}\left(1-\lambda^{i}\right)\right)^{k} \phi^{2 k}(1-\phi)^{n-2 k} \\
& -2 \sum_{k=0}^{\left\lfloor\frac{n-1}{2}\right\rfloor} \frac{n !}{k !(k+1) !(n-1-2 k) !}\left(\lambda^{i}\left(1-\lambda^{i}\right)\right)^{k+1} \phi^{2 k+1}(1-\phi)^{n-1-2 k} .
\end{aligned}
$$

For an odd $n$, note that

$$
\begin{aligned}
& \sum_{k=0}^{\left\lfloor\frac{n}{2}\right\rfloor} \frac{n !}{(k !)^{2}(n-2 k) !}\left(\lambda^{i}\left(1-\lambda^{i}\right)\right)^{k} \phi^{2 k}(1-\phi)^{n-2 k} \\
& \quad=\sum_{k=0}^{\frac{n-1}{2}}\left(1+\frac{2 k}{n-2 k}\right) \frac{(n-1) !}{(k !)^{2}(n-1-2 k) !}\left(\lambda^{i}\left(1-\lambda^{i}\right)\right)^{k} \phi^{2 k}(1-\phi)^{n-2 k} \\
& =(1-\phi) \sum_{k=0}^{\frac{n-1}{2}} \frac{(n-1) !}{(k !)^{2}(n-1-2 k) !}\left(\lambda^{i}\left(1-\lambda^{i}\right)\right)^{k} \phi^{2 k}(1-\phi)^{n-1-2 k} \\
& \quad+2 \phi \sum_{k=0}^{\frac{n-1}{2}-1} \frac{(n-1) !}{k !(k+1) !(n-2-2 k) !}\left(\lambda^{i}\left(1-\lambda^{i}\right)\right)^{k+1} \phi^{2 k+1}(1-\phi)^{n-2-2 k} .
\end{aligned}
$$

Inserting this fact and simplifying terms yield

$$
\begin{aligned}
& T\left(\phi, n \mid \lambda_{A}=\lambda^{i}\right)-T\left(\phi, n+1 \mid \lambda_{A}=\lambda^{i}\right) \\
& =\sum_{k=0}^{\frac{n-1}{2}} \frac{(n-1) !}{(k !)^{2}(n-1-2 k) !}\left(\lambda^{i}\left(1-\lambda^{i}\right)\right)^{k} \phi^{2 k+1}(1-\phi)^{n-1-2 k} \\
& \quad+2 \lambda^{i}\left(1-\lambda^{i}\right) \sum_{k=0}^{\frac{n-1}{2}-1} \frac{(n-1) !}{k !(k+1) !(n-2-2 k) !}\left(\lambda^{i}\left(1-\lambda^{i}\right)\right)^{k} \phi^{2 k+1}(1-\phi)^{n-1-2 k} \\
& \quad-2 \lambda^{i}\left(1-\lambda^{i}\right) \sum_{k=0}^{\frac{n-1}{2}} \frac{n !}{k !(k+1) !(n-1-2 k) !}\left(\lambda^{i}\left(1-\lambda^{i}\right)\right)^{k} \phi^{2 k+1}(1-\phi)^{n-1-2 k}>0 .
\end{aligned}
$$

The same exact argument follows for $n$ even. Finally, similar arguments for $\lim _{n \rightarrow \infty} \alpha^{*}(n)=0$ show $\lim _{n \rightarrow \infty} \phi^{*}(n)=0$.

Proof of Lemma 7. This uses the exact lines in the proof of Lemma 4, except that we replace $\alpha_{A}(n)$ with $\lambda_{A} \phi^{*}(n)$, and $\alpha_{B}(n)$ with $\lambda_{B} \phi^{*}(n)$.

Proof of Proposition 6. The existence of a unique symmetric BNE follows from Proposition 5, Lemma 5, and the observation that $\lim _{n \rightarrow \infty} \stackrel{\underline{c}(n)=0}{0}$ by part (i) of Lemma 3 . Now, we prove the remainder of the proposition. 
(a). Observe that $X_{A} \sim \operatorname{Poisson}\left(\lambda_{A} M^{U}\right)$ and $X_{B} \sim \operatorname{Poisson}\left(\left(1-\lambda_{A}\right) M^{U}\right)$. Now, for $\lambda_{A}=\lambda^{i}$,

$$
\begin{aligned}
\operatorname{Pr} & \left\{A \text { wins } \mid \lambda^{i}\right\}>\frac{1}{2} \\
& \Leftrightarrow \operatorname{Pr}\left\{X_{A}>X_{B}\right\}+\frac{1}{2} \operatorname{Pr}\left\{X_{A}=X_{B}\right\}>\operatorname{Pr}\left\{X_{B}>X_{A}\right\}+\frac{1}{2} \operatorname{Pr}\left\{X_{B}=X_{A}\right\} \\
& \Leftrightarrow \operatorname{Pr}\left\{X_{A}>X_{B}\right\}>\operatorname{Pr}\left\{X_{B}>X_{A}\right\} \\
& \Leftrightarrow \sum_{j=0}^{\infty} \sum_{k=j+1}^{\infty} f\left(j \mid\left(1-\lambda^{i}\right) M^{U}\right) f\left(k \mid \lambda^{i} M^{U}\right)>\sum_{j=0}^{\infty} \sum_{k=j+1}^{\infty} f\left(j \mid \lambda^{i} M^{U}\right) f\left(k \mid\left(1-\lambda^{i}\right) M^{U}\right) .
\end{aligned}
$$

Now, suppose $\lambda^{i}>\frac{1}{2}$ and consider the following sequence of implications where $k \neq j$ :

$$
\begin{aligned}
\lambda^{i}>\frac{1}{2} & \Leftrightarrow \lambda^{i}>\left(1-\lambda^{i}\right) \Leftrightarrow\left(\lambda^{i}\right)^{k-j}>\left(1-\lambda^{i}\right)^{k-j} \\
& \Leftrightarrow\left(\lambda^{i} M^{U}\right)^{k}\left(\left(1-\lambda^{i}\right) M^{U}\right)^{j}>\left(\lambda^{i} M^{U}\right)^{j}\left(\left(1-\lambda^{i}\right) M^{U}\right)^{k} \\
& \Leftrightarrow f\left(j \mid\left(1-\lambda^{i}\right) M^{U}\right) f\left(k \mid \lambda^{i} M^{U}\right)>f\left(j \mid \lambda^{i} M^{U}\right) f\left(k \mid\left(1-\lambda^{i}\right) M^{U}\right) \\
& \Leftrightarrow \sum_{j=0}^{\infty} \sum_{k=j+1}^{\infty} f\left(j \mid\left(1-\lambda^{i}\right) M^{U}\right) f\left(k \mid \lambda^{i} M^{U}\right)>\sum_{j=0}^{\infty} \sum_{k=j+1}^{\infty} f\left(j \mid \lambda^{i} M^{U}\right) f\left(k \mid\left(1-\lambda^{i}\right) M^{U}\right) .
\end{aligned}
$$

The same argument shows that $\operatorname{Pr}\left\{A\right.$ wins $\left.\mid \lambda^{i}\right\}<\frac{1}{2}$ if and only if $\lambda^{i}<\frac{1}{2}$, and $\operatorname{Pr}\left\{A\right.$ wins $\left.\mid \lambda^{i}\right\}=\frac{1}{2}$ if and only if $\lambda^{i}=\frac{1}{2}$.

(b), (c). Here, we first prove part (c). The fact that $m_{A}^{U}=\lambda_{A} M^{U}$, and $m_{B}^{U}=\lambda_{B} M^{U}$ follows from Lemma 7. Moreover, one can easily show as in the proof of part (b) of Proposition 3 that $\Phi^{U}(M \mid \lambda, \theta)$ is strictly decreasing in $M$; $\Phi^{U}(0 \mid \lambda, \theta)=1$; and (3) $\lim _{M \rightarrow \infty} \Phi^{U}(M \mid \lambda, \theta)=0$. Hence, there exists a unique $M^{U} \in(0, \infty)$ that solves $\Phi^{U}(M \mid \lambda, \theta)=2 c$. From here, it also follows that $M^{U}$ is strictly decreasing in $c$, and becomes arbitrarily large as $c \rightarrow 0$. Together with part (a), this proves part (b).

Proof of Proposition 7. Suppose (4) and (5) hold so that a symmetric totally-mixed strategy BNE obtains in either informational setting. Each part is proven in turn.

(a). Suppose $n<\infty$. Since for $\lambda_{A}=\lambda^{i}, C^{I}\left(n \mid \lambda_{A}=\lambda^{i}\right)=2 n c \alpha^{*}(n)$ by Proposition 1, and $C^{U}\left(n \mid \lambda_{A}=\lambda^{i}\right)=n c \phi^{*}(n)$ by Proposition 4 , it suffices to show that $\alpha^{*}(n)>\frac{\phi^{*}(n)}{2}$. Define $\widehat{\phi}=\frac{\phi}{2}$ and $\widehat{P}_{A}\left(\widehat{\phi} \mid n, \lambda_{A}=\lambda^{i}\right) \equiv P_{A}\left(\lambda^{i} 2 \widehat{\phi},\left(1-\lambda^{i}\right) 2 \widehat{\phi}, n\right)$. Then,

$$
\begin{aligned}
\widehat{P}_{A}\left(\widehat{\phi} \mid n, \lambda_{A}=\lambda^{i}\right)= & \sum_{k=0}^{\left\lfloor\frac{n-1}{2}\right\rfloor}\left(\begin{array}{c}
n-1 \\
k, k, n-1-2 k
\end{array}\right) 2^{2 k}\left(\lambda^{i}\left(1-\lambda^{i}\right)\right)^{k} \widehat{\phi}^{2 k}(1-2 \widehat{\phi})^{n-1-2 k} \\
& +\sum_{k=0}^{\left\lfloor\frac{n-2}{2}\right\rfloor}\left(\begin{array}{c}
n-1 \\
k, k+1, n-2-2 k
\end{array}\right) 2^{2 k+1}\left(\lambda^{i}\right)^{k}\left(1-\lambda^{i}\right)^{k+1} \widehat{\phi}^{2 k+1}(1-2 \widehat{\phi})^{n-2-2 k} .
\end{aligned}
$$

Since $2 \lambda^{i}\left(2\left(1-\lambda^{i}\right)\right)<1$, we have

$$
\begin{aligned}
\widehat{P}_{A}\left(\widehat{\phi} \mid n, \lambda_{A}=\lambda^{i}\right)< & \sum_{k=0}^{\left\lfloor\frac{n-1}{2}\right\rfloor}\left(\begin{array}{c}
n-1 \\
k, k, n-1-2 k
\end{array}\right) \widehat{\phi}^{2 k}(1-2 \widehat{\phi})^{n-1-2 k} \\
& +2\left(1-\lambda^{i}\right) \sum_{k=0}^{\left\lfloor\frac{n-2}{2}\right\rfloor}\left(\begin{array}{c}
n-1 \\
k, k+1, n-2-2 k
\end{array}\right) \widehat{\phi}^{2 k+1}(1-2 \widehat{\phi})^{n-2-2 k} .
\end{aligned}
$$

Moreover, since $\sum_{i=1}^{r} \theta^{i} \lambda^{i}=\frac{1}{2}$ and $\sum_{i=1}^{r} \theta^{i} \lambda^{i} 2\left(1-\lambda^{i}\right)<\frac{1}{2}$, it follows that

$$
\sum_{i=1}^{r} \theta^{i} \lambda^{i} \widehat{P}_{A}\left(\widehat{\phi} \mid n, \lambda_{A}=\lambda^{i}\right)=\frac{1}{2} Q(2 \widehat{\phi}, n)<\frac{1}{2} P(\widehat{\phi}, n) .
$$

Finally, Lemma 2 and the proof of Proposition 5 established that both $P(\hat{\phi}, n)$ and $Q(2 \widehat{\phi}, n)$ are decreasing in $\widehat{\phi}$, implying that $\alpha^{*}>\frac{\phi^{*}}{2}$ where $\frac{1}{2} P\left(\alpha^{*}, n\right)-c=0$ and $\frac{1}{2} Q\left(2\left(\frac{\phi^{*}}{2}\right), n\right)-c=0$. 
For $n \rightarrow \infty$, we have $2 n \alpha^{*}(n) \rightarrow M^{I}$ and $n \phi^{*}(n) \rightarrow M^{U}$ by definition; and hence $C^{I}\left(\phi^{*}(n), n \mid \lambda_{A}=\lambda^{i}\right) \rightarrow c M^{I}$ and $C^{U}\left(\phi^{*}(n), n \mid \lambda_{A}=\lambda^{i}\right) \rightarrow c M^{U}$, where, the limit turnouts under the informed and informed regimes, $M^{I}$ and $M^{U}$ satisfy $\Phi^{I}\left(\frac{M}{2}, \frac{M}{2}\right)=2 c$ and $\Phi^{U}(M \mid \lambda, \theta)=2 c$, respectively. Since, from the proofs of Propositions 3 and 6 , both $\Phi^{I}\left(\frac{M}{2}, \frac{M}{2}\right)$ and $\Phi^{U}(M \mid \lambda, \theta)$ are strictly decreasing in $M, \Phi^{I}(0,0)=\Phi^{U}(0 \mid \lambda, \theta)=1$, and $\sum_{i=1}^{r} \theta^{i} \lambda^{i}=\frac{1}{2}$ and $\sum_{i=1}^{r} \theta^{i} \lambda^{i} 2\left(1-\lambda^{i}\right)<\frac{1}{2}$ by definition, a similar comparison to the case with $n<\infty$ implies $M^{I}>M^{U}$.

(b). Suppose $n<\infty$. From Propositions 1 and 4 , it is obvious that $B^{I}\left(\phi, n \mid \lambda_{A}=\lambda^{i}\right)<B^{U}\left(\phi, n \mid \lambda_{A}=\lambda^{i}\right)$, and hence $\bar{B}^{I}(\theta, \lambda, n)<\bar{B}^{U}(\theta, \lambda, n)$. This result also holds for $n \rightarrow \infty$, because

$$
\frac{B^{I}\left(\phi, n \mid \lambda_{A}=\lambda^{i}\right)}{B^{U}\left(\phi, n \mid \lambda_{A}=\lambda^{i}\right)} \rightarrow \frac{\frac{1}{2}}{\operatorname{Pr}\left\{A \text { wins } \mid \lambda_{A}=\lambda^{i}\right\} \lambda^{i}+\operatorname{Pr}\left\{B \text { wins } \mid \lambda_{A}=\lambda^{i}\right\}\left(1-\lambda^{i}\right)}<1
$$

by Proposition 4 .

(c). It directly follows from part (a) and (b).

Proof of Proposition 8. Let $\theta$ be a symmetric distribution over a fixed support $\lambda$, and $\phi^{*}(\theta)$ be the equilibrium voting probability, which satisfies (13). Decomposing the summation for some $i_{0} \leqslant\left\lfloor\frac{r+1}{2}\right\rfloor$, we re-write (13)

$$
\sum_{i=1}^{i_{0}-1} \theta^{i} T\left(\phi^{*}(n), n \mid \lambda_{A}=\lambda^{i}\right)+\left(\frac{1}{2}-\sum_{i \neq i_{0}}^{\left\lfloor\frac{r+1}{2}\right\rfloor} \theta^{i}\right) T\left(\phi^{*}(n), n \mid \lambda_{A}=\lambda^{i_{0}}\right)+\sum_{i=i_{0}+1}^{\left\lfloor\frac{r+1}{2}\right\rfloor} \theta^{i} T\left(\phi^{*}(n), n \mid \lambda_{A}=\lambda^{i}\right)=c .
$$

Totally differentiating both sides of (A.7) w.r.t. some $\theta_{j}\left(j \neq i_{0}\right.$, and $\left.j \leqslant\left\lfloor\frac{r+1}{2}\right\rfloor\right)$, we obtain

$$
\frac{\partial}{\partial \theta_{j}} \phi^{*}(\theta)=\frac{T\left(\phi^{*}(n), n \mid \lambda_{A}=\lambda^{i_{0}}\right)-T\left(\phi^{*}(n), n \mid \lambda_{A}=\lambda^{j}\right)}{\theta_{j} T_{\phi}\left(\phi^{*}(\theta), n \| \lambda_{A}=\lambda^{j}\right)+\left(\frac{1}{2}-\sum_{i \neq i_{0}}^{\left\lfloor\frac{r+1}{2}\right\rfloor} \theta^{i}\right) T_{\phi}\left(\phi^{*}(\theta), n \mid \lambda_{A}=\lambda^{i_{0}}\right)} .
$$

To sign $\phi_{j}^{*}(\theta)$, first recall from the proof of Proposition 5 that $\frac{\partial}{\partial \phi} T($.) $<0$. Moreover, it is easy to verify from (12) that $T\left(\phi^{*}(n), n \mid \lambda_{A}=\lambda^{i}\right)$ is increasing in $\lambda^{i}$ for $\lambda^{i}<\frac{1}{2}$, stationary at $\lambda^{i}=\frac{1}{2}$, and decreasing in $\lambda^{i}$ for $\lambda^{i}>\frac{1}{2}$. Thus, for any $j<i_{0}$, we have $T\left(\phi^{*}(n), n \mid \lambda_{A}=\lambda^{i_{0}}\right)-T\left(\phi^{*}(n), n \mid \lambda_{A}=\lambda^{j}\right)>0$, which implies $\frac{\partial}{\partial \theta_{j}} \phi^{*}(\theta)<0$. A similar argument for any $i_{0}<j \leqslant$ $\left\lfloor\frac{r+1}{2}\right\rfloor$ shows that $\frac{\partial}{\partial \theta_{j}} \phi^{*}(\theta)>0$.

These two observations reveal that if $\theta^{\prime}$ and $\theta$ are two symmetric distributions such that $\theta^{\prime}$ is a mean-preserving spread over $\theta$ as defined in Section 6 , then $\phi^{*}\left(\theta^{\prime}\right) \leqslant \phi^{*}(\theta)$, proving part (a).

To prove part (b), we recall the definition $\bar{B}(\theta, \lambda, n)$ in Section 5, and w.l.o.g., use a similar decomposition as in part (a). This implies

$$
\bar{B}(\theta, \lambda, \phi, n)=\sum_{i=1}^{i_{0}-1} \theta^{i} B\left(\phi, n \mid \lambda_{A}=\lambda^{i}\right)+\left(\frac{1}{2}-\sum_{i \neq i_{0}}^{\left\lfloor\frac{r+1}{2}\right\rfloor} \theta^{i}\right) B\left(\lambda^{i_{0}}, \phi, n\right)+\sum_{i=i_{0}+1}^{\left\lfloor\frac{r+1}{2}\right\rfloor} \theta^{i} B\left(\phi, n \mid \lambda_{A}=\lambda^{i}\right)
$$

where

$$
B\left(\phi, n \mid \lambda_{A}=\lambda^{i}\right)=\operatorname{Pr}\left\{A \text { wins } \mid \lambda_{A}=\lambda^{i}\right\} \lambda^{i} n+\operatorname{Pr}\left\{B \text { wins } \mid \lambda_{A}=\lambda^{i}\right\}\left(1-\lambda^{i}\right) n .
$$

Moreover, we have

$$
\begin{aligned}
& \operatorname{Pr}\left\{A \text { wins } \mid \lambda_{A}=\lambda^{i}\right\}=\sum_{k=0}^{n} \sum_{k^{\prime}=k+1}^{n}\left(\begin{array}{c}
n \\
k, k^{\prime}, n-k-k^{\prime}
\end{array}\right)\left(\lambda^{i} \phi^{*}(\theta)\right)^{k^{\prime}}\left(\left(1-\lambda^{i}\right) \phi^{*}(\theta)\right)^{k}\left(1-\phi^{*}(\theta)\right)^{n-k-k^{\prime}}, \\
& \operatorname{Pr}\left\{B \text { wins } \mid \lambda_{A}=\lambda^{i}\right\}=\operatorname{Pr}\left\{A \text { wins } \mid \lambda_{A}=1-\lambda^{i}\right\} .
\end{aligned}
$$

Inserting (A.11) into (A.10), and then into (A.9) yields

$$
\overline{\bar{B}}\left(\theta, \phi^{*}(\theta)\right)=\bar{B}\left(\theta, \lambda, \phi^{*}(\theta), n\right) .
$$

Differentiating w.r.t. some $\theta_{j}, j \in\left\{1, \ldots, i_{0}\right\}$, implies

$$
\frac{\partial}{\partial \theta_{j}} \overline{\bar{B}}\left(\theta, \phi^{*}(\theta)\right)+\frac{\partial}{\phi} \overline{\bar{B}}\left(\theta, \phi^{*}(\theta)\right) \frac{\partial}{\partial \theta_{j}} \phi^{*}(\theta) \text {. }
$$


It is easy to show that $\frac{\partial}{\phi} \overline{\bar{B}}\left(\theta, \phi^{*}(\theta)\right)>0$ because $\frac{\partial}{\phi} B\left(\phi, n \mid \lambda_{A}=\lambda^{i}\right)>0$, and

$$
\frac{\partial}{\partial \theta_{j}} \overline{\bar{B}}\left(\theta, \phi^{*}(\theta)\right) \begin{cases}\geqslant 0 & \text { if } 1 \leqslant j<i_{0}, \\ \leqslant 0 & \text { if } i_{0}<j \leqslant\left\lfloor\frac{r+1}{2}\right\rfloor .\end{cases}
$$

From the proof of part (a), this means for $1 \leqslant j<i_{0}$, while the first term in (A.12) is positive, the second term is negative. However, a tedious algebra shows that it is overall positive. A similar argument shows that the overall sign is negative for $i_{0}<j \leqslant\left\lfloor\frac{r+1}{2}\right\rfloor$. Together these two observations prove part (b).

The last part directly follows from parts (a) and (b).

\section{References}

Acemoglu, D., Robinson, J., 2006. Economic Origins of Dictatorship and Democracy. Cambridge Univ. Press.

Aldrich, J., 1993. Rational choice and turnout. Amer. J. Polit. Sci. 37, 246-278.

Barone, M., 2006. Close elections: Is this a trend? Real Clear Polit., July 17.

Bernhardt, D., Duggan, J., Squintani, F., forthcoming. Private polling in elections and voter welfare. J. Econ. Theory

Billingsley, P., 1995. Probability and Measure. Wiley.

Borgers, T., 2004. Costly voting. Amer. Econ. Rev. 94, 57-66.

Campbell, C., 1999. Large electorates and decisive minorities. J. Polit. Economy 107, 1199-1217.

Durand, C., Blais, A., Larochelle, M., 2004. The polls in the 2002 French Presidential Elections: An autopsy. Public Opinion Quart. 68 (4), 602-622.

Durand, C., Blais, A., Vachon, S., 2001. The late campaign swing or a failure of the polls? The case of the 1998 Quebec election. Public Opinion Quart. 65, $108-123$.

Dutta, B., Jackson, M., Le Breton, M., 2001. Strategic candidacy and voting procedures. Econometrica 69, 1013-1037.

Feddersen, T., Pesendorfer, W., 1997. Voting behavior and information aggregation in elections with private information. Econometrica 65, $1029-1058$.

Goeree, J., Grosser, J., 2007. False consensus voting and welfare reducing polls. Econ. Theory 31 (1), 51-68.

Hansen, S., Palfrey, T., Rosenthal, H., 1987. The Downsian model of electoral participation: Formal theory and empirical analysis of the constituency size effect. Public Choice 52, 15-33.

Jowell, R., Hedges, B., Lynn, P., Farrant, G., Heath , A., 1993. The 1992 British Election: The failure of the polls. Public Opinion Quart. 57, $238-263$.

Klor, E., Winter, E., 2006. On public opinion polls and voters' turnout. Working paper, The Hebrew University of Jerusalem.

Krasa, S., Polborn, M., 2009. Is mandatory voting better than voluntary voting? Games Econ. Behav. 66, 275-291.

Ledyard, J., 1984. The pure theory of large two-candidate elections. Public Choice 44, 7-41.

Levine, D., Palfrey, T., 2007. The paradox of voter participation? A laboratory study. Amer. Polit. Sci. Rev. 101, $143-158$.

Martinelli, C., 2006. Would rational voters acquire costly information? J. Econ. Theory 129, $225-251$.

Myerson, R., 1998. Population uncertainty and poisson games. Int. J. Game Theory 27, 375-392.

Myerson, R., 2000. Large poisson games. J. Econ. Theory 94, 7-45.

Osborne, M., Rosenthal, J., Turner, M., 2000. Meetings with costly participation. Amer. Econ. Rev. 90, $927-943$.

Ottaviani, M., Sorensen, P., 2006. The strategy of professional forecasting. J. Finan. Econ. 81 (2), 441-466.

Palfrey, T., Rosenthal, H., 1983. A strategic calculus of voting. Public Choice 41, 7-53.

Palfrey, T., Rosenthal, H., 1985. Voter participation and strategic uncertainty. Amer. Polit. Sci. Rev. 79, 62-78.

Razin, R., 2003. Signalling and election motivations in a voting model with common values and responsive candidates. Econometrica 71 (4), 1083-1119.

Sudman, S., 1986. Do exit polls influence voting behavior. Public Opinion Quart. 50, 331-339.

Taylor, C., Yildirim, H., 2008. A unified analysis of rational voting with private values and cost uncertainty. Working paper, Duke University.

West, D., 1991. Polling effects in election campaigns. Polit. Behav. 13, 151-163. 